\title{
For a Few Dollars More: Verified Fine-Grained Algorithm Analysis Down to LLVM
}

\author{
MAXIMILIAN P. L. HASLBECK, Technische Universität München \\ PETER LAMMICH, University of Twente
}

\begin{abstract}
We present a framework to verify both, functional correctness and (amortized) worst-case complexity of practically efficient algorithms. We implemented a stepwise refinement approach, using the novel concept of resource currencies to naturally structure the resource analysis along the refinement chain, and allow a fine-grained analysis of operation counts. Our framework targets the LLVM intermediate representation. We extend its semantics from earlier work with a cost model. As case studies, we verify the amortized constant time push operation on dynamic arrays and the $O(n \log n)$ introsort algorithm, and refine them down to efficient LLVM implementations. Our sorting algorithm performs on par with the state-of-the-art implementation found in the GNU C++ Library, and provably satisfies the complexity required by the $\mathrm{C}++$ standard.
\end{abstract}

CCS Concepts: • Theory of computation $\rightarrow$ Logic and verification; Separation logic; Program semantics; Program verification;

Additional Key Words and Phrases: Algorithm analysis, program verification, refinement, Isabelle/HOL

\section{ACM Reference format:}

Maximilian P. L. Haslbeck and Peter Lammich. 2022. For a Few Dollars More: Verified Fine-Grained Algorithm Analysis Down to LLVM. ACM Trans. Program. Lang. Syst. 44, 3, Article 14 (July 2022), 36 pages.

https://doi.org/10.1145/3486169

\section{INTRODUCTION}

In general, not only correctness, but also the complexity of algorithms is important. While it is obvious that the performance observed during experiments is essential to solve practical problems efficiently, also the theoretical worst-case complexity of algorithms is crucial: a good worst-case complexity avoids timing regressions when hitting worst-case input, and, even more important, prevents denial of service attacks that intentionally produce worst-case scenarios to overload critical computing infrastructure.

For example, the $\mathrm{C}++$ standard requires implementations of std::sort to have worst-case complexity $O(n \log n)$ [8]. Note that this rules out quicksort [16], which is very fast in practice, but has

This work was supported by the DFG Koselleck grant NI 491/16-1 "Verifizierte Algorithmenanalyse" and the DFG grant LA 3292/1 "Verifizierte Model Checker".

Authors' addresses: M. P. L. Haslbeck, Technische Universität München, Arcisstraße 21, 80333 München, Munich, Germany; email: haslbema@in.tum.de; P. Lammich, University of Twente, Drienerlolaan 5, 7522 NB, Enschede, Netherlands; email: p.lammich@utwente.nl.

Permission to make digital or hard copies of all or part of this work for personal or classroom use is granted without fee provided that copies are not made or distributed for profit or commercial advantage and that copies bear this notice and the full citation on the first page. Copyrights for components of this work owned by others than the author(s) must be honored. Abstracting with credit is permitted. To copy otherwise, or republish, to post on servers or to redistribute to lists, requires prior specific permission and/or a fee. Request permissions from permissions@acm.org.

(C) 2022 Copyright held by the owner/author(s). Publication rights licensed to ACM.

0164-0925/2022/07-ART14 \$15.00

https://doi.org/10.1145/3486169 
quadratic worst-case complexity. Nevertheless, the widely used standard library LLVM libc++ [27] only recently stopped using quicksort. ${ }^{1}$

A practically efficient sorting algorithm with $O(n \log n)$ worst-case complexity is Musser's introsort [30]. It combines quicksort with the $O(n \log n)$ heapsort algorithm, which is used as fallback when the quicksort recursion depth exceeds a certain threshold. It allows to implement standardcompliant, practically efficient sorting algorithms. Introsort is implemented by, e.g., the GNU C++ Library $(l i b s t d c++)$ [10] and by libc++ [27] since version 14 .

In this article, we present techniques to formally verify both, correctness and worst-case complexity of practically efficient implementations. Our approach seamlessly works for both, standard and amortized analysis. We build on two previous lines of research by the authors.

On the one hand, we have the Isabelle Refinement Framework [26], which allows for a modular top-down verification approach. It utilizes stepwise refinement to separate the different aspects of an efficient implementation, such as algorithmic idea and low-level optimizations. It provides a nondeterminism monad to formalize programs and refinements, and the Sepref tool to automate canonical data refinement steps. Its recent LLVM back end [22] allows to verify algorithms with competitive performance compared to (unverified) highly optimized $\mathrm{C} / \mathrm{C}++$ implementations. The Refinement Framework has been used to verify the functional correctness of an implementation of introsort that performs on par with libstdc++'s implementation [24].

On the other hand, we already have extended the Refinement Framework to reason about complexity [14]. However, the cost model used there limits the natural structuring of the cost analysis in refinement proofs. Moreover, it only supports the Imperative HOL back end [23], which generates functional code that is inherently less efficient than imperative code.

This article extends our conference paper [15] by adding amortized analysis and a case study on dynamic arrays, complexity analysis of string sorting, and more in-depth explanations of the design choices of our framework. We also make the article more self-contained by including material from [14]. Our main contributions are.

- We present a generalized nondeterminism monad with resource cost, apply it to resource functions to model fine-grained currencies (Section 2), and show how they can be used to naturally structure refinement.

- We extend the LLVM back end [22] with a cost model, and amend its basic reasoning infrastructure (Section 3).

- We extend the Sepref tool (Section 4) to synthesize executable imperative code in LLVM, together with a proof of correctness and complexity.

- We show how to integrate the analysis of amortized data structures with our refinement approach (Section 5).

- We extend the verification of introsort to also show a worst-case complexity of $O(n \log n)$, thus meeting the $\mathrm{C}++11$ stdlib specification [8] (Section 6). Our methodology also works for sorting data (e. g., strings) with a comparison operation that does not have constant running time. The performance of our implementation is still on par with libstdc++. We believe that this is the first time that both, correctness and complexity of a sorting algorithm have been formally verified down to a competitive implementation.

Our formalization is available at https://www21.in.tum.de/ haslbema/llvm-time.

\section{SPECIFICATION OF ALGORITHMS WITH RESOURCES}

We use the formalism of monads [35] to elegantly specify programs with resource usage. We first describe a framework that works for a very generic notion of resource, and then instantiate it with

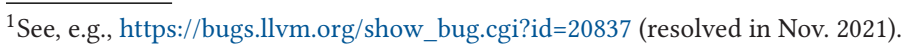


resource functions, which model resources of different currencies. We then describe a refinement calculus and show how currencies can be used to structure stepwise refinement proofs. Finally, we report on automation and discuss alternatives to our modeling of programs with resources.

In this section, we consider purely functional programs. In Section 4, these will be refined to imperative programs.

\subsection{Nondeterministic Computations With Resources}

Let us examine the features we require for our computation model.

First, we want to specify programs by their desired properties, without having to fix a concrete implementation. In general, those programs have more than one correct result for the same input. Consider, e.g., sorting a list of pairs of numbers by the first element. For the input $[(1,2),(2,2),(1,3)]$, both $[(1,2),(1,3),(2,2)]$ and $[(1,3),(1,2),(2,2)]$ are valid results. Formally, this is modeled as a set of possible results. When we later fix an implementation, the set of possible results may shrink. For example, the (stable) insertion sort algorithm always returns the list $[(1,2),(1,3),(2,2)]$. We say that insertion sort refines our specification of sorting.

Second, we want to define recursion by a standard fixed-point construction over a flat lattice. The bottom of this lattice must be a dedicated element, which we call fail. It represents a computation that may not terminate.

Finally, we want to model the resources required by a computation. For nondeterministic programs, these may vary depending on the nondeterministic choices made during the computation. As we model computations by their possible results, rather than by the exact path in the program that leads to the result, we also associate resource cost with possible results. When more than one computation path leads to the same result, we take the supremum of the used resources. The notion of refinement is now extended to a subset of results that are computed using less resources.

We now formalize the above intuition: the type

$$
(\alpha, \gamma) \text { NREST }=\text { fail } \mid \text { res }(\alpha \rightarrow \gamma \text { option })
$$

models a nondeterministic computation with results of type $\alpha$ and resources of type $\gamma^{2}$ That is, a computation is either fail, or res $M$, where $M$ is a partial function from possible results to resources.

Example 2.1. The computation res $[a \mapsto 5, b \mapsto 3]$ either returns $a$ using 5 resources, or $b$ using 3 resources. Here, the notation $\left[a_{1} \mapsto t_{1}, \ldots, a_{n} \mapsto t_{n}\right]$ defines a function mapping each $a_{i}$ to Some $t_{i}$, and any other argument to None.

We define spec $\Phi T$ as a computation of any result $r$ that satisfies $\Phi r$ using $T r$ resources: spec $\Phi T=\operatorname{res}(\lambda r$. if $\Phi r$ then Some $(T r)$ else None). By abuse of notation, we write spec $x t$ for spec $(\lambda r . r=x)\left(\lambda_{-} . t\right)$.

Based on an ordering on the resources $\gamma$, we define the refinement ordering on NREST, by first lifting the ordering to option with None as the bottom element, then pointwise to functions and finally to $(\alpha, \gamma)$ NREST, setting fail as the top element. This matches the intuition of refinement: $m \leq m^{\prime}$ reads as $m$ refines $m^{\prime}$, i.e., $m$ has less possible results than $m^{\prime}$, computed with less resources.

We require the resources $\gamma$ to have a complete lattice structure, such that we can form suprema over the (possibly infinitely many) paths that lead to the same result. Then, also NREST with the refinement ordering forms a complete lattice. The top element is fail, it satisfies no specification. The bottom element is res ( $\lambda_{-}$. None), it satisfies all specifications, but has no implementation.

${ }^{2}$ The name NREST abbreviates Nondeterministic RESult with Time, and has been inherited from our earlier formalizations. 
Moreover, when sequentially composing computations, we need to add up the resources. This naturally leads to a monoid structure $(\gamma, 0,+)$, where 0 , intuitively, stands for no resources. We call such types $\gamma$ resource types, if they have a complete lattice and monoid structure. Note that, in an earlier iteration of this work [14], the resource type was fixed to extended natural numbers (enat $=\mathbb{N} \cup\{\infty\}$ ), measuring the resource consumption with a single number. Also note that ( $\alpha$, unit) NREST is isomorphic to our original nondeterministic result monad without resources [26].

If $\gamma$ is a resource type, so is $\eta \rightarrow \gamma$. Intuitively, such resources consist of coins of different resource currencies $\eta$, the amount of coins being measured by $\gamma .{ }^{3}$

If not indicated otherwise, we use the resource type ecost $=$ string $\rightarrow$ enat, i.e., we have currencies described by a string, whose amount is measured by extended natural numbers, where $\infty$ models arbitrary resource usage. Note that, while the resource type string $\rightarrow$ enat guides intuition, most of our theory works for general resource types of the form $\eta \rightarrow \gamma$ or even just $\gamma$.

We define the function $\$_{s} n$ to be the resource function that uses $n$ coins of the currency $s$, where $n$ is of type enat, and $s$ is of type string. We write $\$_{s}$ as shortcut for $\$_{s} 1$.

Example 2.2. A program that sorts a list in $O\left(n^{2}\right)$ can be specified by:

$$
\text { sort }_{\text {spec }} x s=\operatorname{spec}\left(\lambda x s^{\prime} \text {. sorted } x s^{\prime} \wedge \text { mset } x s^{\prime}=\text { mset } x s\right)\left(\lambda_{-} . \$_{q}|x s|^{2}+\$_{c}\right)
$$

That is, a list $x s$ can result in any sorted list $x s^{\prime}$ with the same elements, and the computation takes (at most) quadratically many $q$ coins in the list length, and one c coin, independently of the list length. Intuitively, the $q$ and $c$ coins represent the constant factors of an algorithm that implements that specification and are later elaborated by exchanging them into several coins of more finegrained currencies, corresponding to the concrete operations in the algorithm, e.g., comparisons and memory accesses. Abstract currencies like $q$ and conly "have value" if they can be exchanged to meaningful other currencies, and finally pay for the resource costs of a concrete implementation.

\subsection{Atomic Operations and Control Flow}

In order to conveniently model actual computations, we define some combinators. The elapse $m t$ combinator adds the (constant) resources $t$ to all results of $m$ :

$$
\begin{aligned}
& \text { elapse }::(\alpha, \gamma) \text { NREST } \rightarrow \gamma \rightarrow(\alpha, \gamma) \text { NREST } \\
& \text { elapse fail } t=\text { fail } \\
& \text { elapse }(\text { res } M) t=\operatorname{res}(\lambda x \text {. case } M x \text { of None } \Rightarrow \text { None } \\
& \left.\qquad \mid \text { Some } t^{\prime} \Rightarrow \text { Some }\left(t+t^{\prime}\right)\right)
\end{aligned}
$$

The program ${ }^{4}$ return $x$ computes the single result $x$ without using any resources:

$$
\begin{aligned}
& \text { return }:: \alpha \rightarrow(\alpha, \gamma) \text { NREST } \\
& \text { return } x=\operatorname{res}[x \mapsto 0]
\end{aligned}
$$

The combinator bind $m f$ models the sequential composition of computations $m$ and $f$, where $f$ may depend on the result of $m$ :

$$
\begin{aligned}
& \text { bind }::(\alpha, \gamma) \text { NREST } \rightarrow(\alpha \rightarrow(\beta, \gamma) \text { NREST }) \rightarrow(\beta, \gamma) \text { NREST } \\
& \text { bind fail } f=\text { fail } \\
& \text { bind }(\operatorname{res} M) f=\operatorname{Sup}\{\text { elapse }(f x) t \mid x t . M x=\text { Some } t\}
\end{aligned}
$$

\footnotetext{
${ }^{3}$ Typically, only finitely many coins have a positive amount.

${ }^{4}$ Note that our shallow embedding makes no formal distinction between syntax and semantics. Nevertheless, we refer to an entity of type NREST, as program to emphasize the syntactic aspect, and as computation to emphasize the semantic aspect.
} 
If the first computation $m$ fails, then also the sequential composition fails. Otherwise, we consider all possible results $x$ with resources $t$ of $m$, invoke $f x$, and add the cost $t$ for computing $x$ to the results of $f x$. The supremum aggregates the cases where $f$ yields the same result, via different intermediate results of $m$, and also makes the whole expression fail if one of the $f x$ fails.

To improve readability of programs, we write $x \leftarrow m$; $f x$ for bind $m(\lambda x . f x)$ and, $m_{1} ; m_{2}$ for bind $m_{1}\left(\lambda_{-} \cdot m_{2}\right)$.

Example 2.3. We now illustrate an effect that stems from our decision to aggregate the resource usage of different computation paths that lead to the same result. Consider the program

res $\left(\lambda n::\right.$ nat. Some $\left.\left(\$_{c} n\right)\right)$; return 0

It first chooses an arbitrary natural number $n$ consuming $n$ coins of currency $c$, and then returns the result 0 . That is, there are arbitrarily many paths that lead to the result 0 , consuming arbitrarily many $c$ coins. The supremum of this is $\infty$, such that the above program is equal to elapse (return 0) $\left(\$_{c} \infty\right)$. Note that none of the computation paths actually attains the aggregated resource usage. We will come back to this in Section 4.5.

Finally, we use Isabelle/HOL's if-then-else and define a recursion combinator rec via a fixedpoint construction [19], to get a complete set of basic combinators. As these combinators also incur cost in the target LLVM, we define resource aware variants:

$$
\begin{aligned}
& \text { if } f_{c} b \text { then } c_{1} \text { else } c_{2}=\text { elapse }\left(r \leftarrow b \text {; if } r \text { then } c_{1} \text { else } c_{2}\right) \$_{\text {if }} \\
& \operatorname{rec}_{c} F x=\text { elapse }\left(\operatorname{rec}\left(\lambda D x . F\left(\lambda x \text {. elapse }(D x) \$_{\text {call }}\right) x\right) x\right) \$_{\text {call }}
\end{aligned}
$$

Here, the guard of if $f_{c}$ is a computation itself, and we consume an additional if coin to account for the conditional branching in the target model. Similarly, every recursive call consumes an additional call coin. Furthermore, we also derive a while combinator:

$$
\text { while } b f s=\operatorname{rec}_{c}\left(\lambda D s \text {. if } f_{c} b \text { then } s \leftarrow f s ; D s \text { else return } s\right) s
$$

While the NREST type allows to specify arbitrary higher-order functions, e.g., a computation that returns a computation (type $\alpha \rightarrow((\beta, \gamma) N R E S T, \gamma) N R E S T)$, in this article we only regard non-nested NREST types. This includes first-order computations like return $:: \alpha \rightarrow(\alpha, \gamma)$ NREST, and combinators like if $::(b o o l, \gamma)$ NREST $\rightarrow(\alpha, \gamma)$ NREST $\rightarrow(\alpha, \gamma)$ NREST $\rightarrow(\alpha, \gamma)$ NREST. This is sufficient to express the programs we are interested in, and closer to the LLVM back end (Section 3), which only supports the if, rec, and while combinators.

\subsection{Specifications}

An NREST program of the form assert $P$; spec $Q T$ is a specification with precondition $P$, postcondition $Q$, and resource usage $T$. Here, an assertion is used to express preconditions of a program. It fails if its condition is not met, and returns unit otherwise:

assert $P=$ if $P$ then return () else fail

A classical Hoare triple for program $m$, with precondition $P$, postcondition $Q$, and a resource usage $t$ (not depending on the result) can be written as a refinement $m \leq \operatorname{assert} P$; spec $Q\left(\lambda_{-} . t\right.$ ).

Example 2.4. Comparison of two list elements at a cost of $t$ can be specified by:

$$
i d x s_{-} c m p_{s p e c} x s i j(t)=\operatorname{asser} t(i<|x s| \wedge j<|x s|) \text {; spec }(x s ! i<x s ! j)\left(\lambda_{-} \cdot t\right)
$$

Here, the term $x s ! i$ is the $i$ th element of list $x s$. Instead of fixing the cost for specifications, we pass them as parameter $t$. This allows us to refine different instances of abstract data types (here lists) by different concrete data structures with different costs. To make bigger programs more readable, we note the cost parameter in parenthesis at the end of the line, as, e.g., in Example 2.7. 
Table 1. This Table Shows the Refinement Steps in the Refinement of list_pushspec Down to an Implementation Using Dynamic Arrays

\begin{tabular}{|c|c|c|c|c|}
\hline Program & Formalism & Currencies & Data Structure & Reference \\
\hline list_push ${ }_{\text {spec }}$ & NREST specification & \$list_push & list & Example 2.5 \\
\hline$d l_{\text {_p}}$ ush $h_{\text {spec }}$ & NREST specification & \$list_push & dynamic list & Example 2.6 \\
\hline$d l \_p u s h$ & NREST program & abstract currencies & dynamic list & Section 5.1 \\
\hline da_push & NREST program & LLVM currencies & dynamic list & Section 5.4 \\
\hline$d a \_p u s h_{\dagger}$ & LLVM program & LLVM currencies & dynamic array & Section 5.4 \\
\hline
\end{tabular}

Example 2.5. Consider the amortized constant time push operation of dynamic arrays. Abstractly, we specify appending an element at the end of a list.

$$
\text { list_push }_{\text {spec }} x s x(t)=\operatorname{spec}[(x s \cdot[x]) \mapsto t]
$$

Here, the term $x s \cdot y s$ denotes appending of two lists and we leave the amount of consumed resource $t$ as a parameter. This specification has no precondition.

As a running example throughout the article, we refine this specification to an LLVM implementation using dynamic arrays. Table 1 lists the most important intermediate steps along the refinement chain: first we refine lists with dynamic lists $\left(d l_{-}\right.$push $\left.h_{\text {spec }}\right)$, then phrase the abstract algorithm ( $\left.d l_{\_} p u s h\right)$, and refine it to only use basic operations (da_push). Finally, we synthesize executable LLVM code ( $\left.d a_{-} p u s h_{\uparrow}\right)$. Note that the NREST-monad is used to model both, specifications and programs. Only in the last step, where imperative data structures are introduced, we switch to (deterministic) LLVM programs. We will come back to this table after we have completed the refinement in Section 5.5.

\subsection{Refinement on NREST}

We have used the refinement ordering to express Hoare triples. Two other applications of refinement are data refinement and currency refinement.

2.4.1 Data Refinement. A typical use-case of refinement is to implement an abstract data type by a concrete data type. For example, we could implement (finite) sets of numbers by sorted distinct lists. We define a refinement relation $R$ between a concrete and an abstract data type. A concrete computation $m$ then refines an abstract computation $m^{\prime}$, if every possible concrete result is related to a possible abstract result. Formally, $m \leq \Downarrow_{D} R m^{\prime}$, where the operator $\Downarrow_{D}$ is defined, for arguments $R$ and $m^{\prime}$, by the following two rules.

$$
\Downarrow_{D} R(\operatorname{res} M)=\operatorname{res}(\lambda c . \operatorname{Sup}\{M a \mid a .(c, a) \in R\}) \quad \Downarrow_{D} R \text { fail }=\text { fail }
$$

Again, we use the supremum to aggregate the costs of all abstract results that are related to a concrete result. As in Example 2.3, this leads to the possibility that the supremum cost is not attained, which we discuss in Section 4.5.

Example 2.6. Recall the example of the dynamic array. We model dynamic arrays $(d a)$ first abstractly by dynamic lists $(d l)$. They consist of a carrier list $c s$ and two numbers $l$ and $c$ representing the length and the capacity of the dynamic list. A list as is refined by a dynamic list $(c s, l, c)$, if the first $l$ elements of $c s$ form the list as. Furthermore, in a valid dynamic list the length is at most the capacity and the capacity is the length of the carrier list. Formally:

$$
((c s, l, c), \text { as }) \in R_{d y n l i s t}^{\text {list }} \longleftrightarrow \text { take } l c s=\text { as } \wedge l \leq c \wedge c=|c s|
$$


Using this representation, we can now specify a push operation on dynamic lists. A push of an element $x$ to a dynamic list $(c s, l, c)$ will result in a valid dynamic list that contains the same elements as before and adds the element $x$ at the end. As the dynamic list may have reached its capacity, it may be necessary to increase the capacity. We can state the intuition in the following NREST specification:

$$
\begin{aligned}
& d l_{-} \text {push }_{\text {spec }}(c s, l, c) x(t)=\operatorname{spec}(\left(c s^{\prime}, l^{\prime}, c^{\prime}\right) . \text { take } l c s^{\prime}=\text { take } l c s \wedge c s^{\prime} ! l=x \\
&\left.\wedge l^{\prime} \leq c^{\prime} \wedge c^{\prime}=\left|c s^{\prime}\right| \wedge l^{\prime}=l+1 \wedge c^{\prime} \geq c\right)\left(\lambda_{-} . t\right)
\end{aligned}
$$

Here, we first only specify the functional correctness, and leave the cost $t$ as a parameter. We already fix that the program has constant cost, independent from the result and the input. The specification requires that the resulting dynamic list contains all the elements as before and adds $x$ at the end. It is not specified whether or how much the carrier list has to increase.

We can now show that the push operation on dynamic lists refines the list_push $_{\text {spec }}$ operation on lists:

$$
\begin{aligned}
& ((c s, l, c), \text { as }) \in R_{d y n l i s t}^{\text {list }} \wedge\left(x, x^{\prime}\right) \in I d \\
& \quad \Longrightarrow d l_{-} \text {push } h_{\text {spec }}(c s, l, c) x(t) \leq \Downarrow_{D} R_{d y n l i s t}^{\text {list }}\left(\text { list_push }_{s p e c} \text { as } x^{\prime}(t)\right)
\end{aligned}
$$

2.4.2 Currency Refinement. In Example 2.4 we have specified how to compare two list elements. We now refine this into a program that first accesses the elements and then compares them.

Example 2.7. We refine idxs_cmp $p_{\text {spec }}\left(\$_{i d x s_{-} c m p}\right)$ from Example 2.4 as follows:

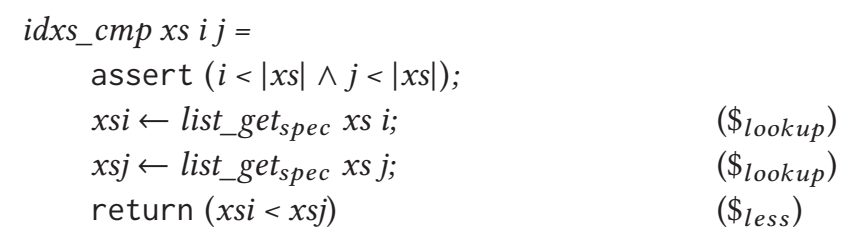

where list_get $t_{\text {spec }} x s i(t)=\operatorname{asser} t(i<|x s|)$; spec $(x s ! i)\left(\lambda_{-} . t\right)$ and return $x(t)$ returns the result $x$ incurring cost $t$.

Note that $i d x s_{-} c m p$ and $i d x s_{-} c m p_{\text {spec }}$ use different, incompatible currency systems. To compare them, we need to exchange coins: one $i d x s \_c m p$ coin will be traded for two lookup coins and one less coin.

To make that happen we introduce the currency refinement $\Downarrow_{C} E m$. Here, for a program $m$ of type $\left(\alpha, \eta_{a} \rightarrow \gamma\right)$ NREST, the exchange rate $E:: \eta_{a} \rightarrow \eta_{c} \rightarrow \gamma$ specifies for each abstract currency $c_{a}:: \eta_{a}$ how many of the coins of the concrete currency $c_{c}:: \eta_{c}$ are needed. Note that, in general, one abstract coin may be exchanged into multiple coins of different currencies. For a resource type $\gamma$ that provides a multiplication operation $(*)$ we define the operator $\Downarrow_{C}$ with the following two rules:

$$
\begin{gathered}
\Downarrow_{C} E(\operatorname{res} M)=\operatorname{res}(\lambda r \text {. case } M r \text { of None } \Rightarrow \text { None } \mid \\
\text { Some } \left.t \Rightarrow \text { Some }\left(\lambda c_{c} \cdot \sum_{c_{a}} t c_{a} * E c_{a} c_{c}\right)\right) \\
\Downarrow_{C} E \text { fail = fail }
\end{gathered}
$$

The refined computation has the same results as the original. To get the amount of a concrete coin $c_{c}$ for some result $r$ with resource function $t$, we sum, over all abstract coins $c_{a}$, the amount of abstract coins needed in the original computation $\left(t c_{a}\right)$ weighted by the exchange rate $\left(E c_{a} c_{c}\right)$.

The sum only makes sense, if there are finitely many abstract coins $c_{a}$ with $t c_{a} * E c_{a} c_{c} \neq 0$. This can be ensured by restricting the resource functions $t$ of the computation to use finitely many 
different coins, or by restricting the exchange rate $E$ accordingly. The latter can be checked syntactically in practice.

Example 2.8. For refining idxs_cmpspec we define an exchange rate that does the correct exchange for currency idxs_cmp and is zero everywhere else. Formally: $E_{1}=$ $\uparrow \downarrow\left[i d x s \_c m p:=\$\right.$ lookup $2+\$$ less $]$. Here, + is lifted to functions in a pointwise manner and $\uparrow \downarrow\left[c_{0}:=t_{0}, \ldots, c_{n}:=t_{n}\right]$ denotes a function that maps the elements $c_{i}$ to $t_{i}$ and all other elements to 0 . We can now prove:

$i d x s_{-}$cmpxs $i j \leq \Downarrow_{C} E_{1}\left(i d x s_{-} c m p_{s p e c} x s i j\left(\$_{i d x s_{-} c m p}\right)\right)$

\subsection{Notation for Refinement}

When considering data refinement, we will often see propositions of the form

$$
\forall x x^{\prime} . P x x^{\prime} \wedge\left(x, x^{\prime}\right) \in R \Longrightarrow f x \leq \Downarrow_{D} S\left(f^{\prime} x\right)
$$

This states that $f$ refines $f^{\prime}$ w.r. t. relation $R$ for the arguments and relation $S$ for the result, if the additional precondition $P$ holds for the arguments. To write those propositions more conveniently, we use the following notation ${ }^{5}$ :

$$
\left(f, f^{\prime}\right) \in[P] R \rightarrow S=\left(\forall x x^{\prime} . P x x^{\prime} \wedge\left(x, x^{\prime}\right) \in R \Longrightarrow f x \leq \Downarrow_{D} S\left(f^{\prime} x^{\prime}\right)\right)
$$

If the precondition is always true, we just write $\left(f, f^{\prime}\right) \in R \rightarrow S$. For the sake of readability, we will identify curried and uncurried functions and write $\left(f, f^{\prime}\right) \in R_{1} \rightarrow \ldots \rightarrow R_{n} \rightarrow S$ for programs with $n$ arguments that are refined by $R_{1}, \ldots, R_{n}$.

The above form of those propositions is called the parametric form. It brings to mind relational parametricity by Wadler [34].

Example 2.9. Using that notation, the refinement from Example 2.6 reads as follows:

$$
\left(d l_{-} \text {push }_{\text {spec }}(t), \text { list_push }_{\text {spec }}(t)\right) \in R_{\text {dynlist }}^{\text {list }} \rightarrow I d \rightarrow R_{\text {dynlist }}^{\text {list }}
$$

That is, if the parameters are related by $R_{d y n l i s t}^{l i s t}$ and the identity relation $I d$, then the result of $d l_{\_} p u s h_{s p e c}$ refines the result of $l i s t_{-} p u s h_{\text {spec }}$ w. r. t. relation $R_{d y n l i s t}^{l i s t}$.

\subsection{Refinement Patterns}

In practice, we encounter certain recurring patterns of refinement, which we describe in this section.

Refinement of Specifications. A common application is to show that a program $m$ satisfies a specification res $Q$, formally $m \leq$ res $Q$. For example, in Section 6.2 we show that the introsort program refines the specification of sorting a slice of a list. Such proofs are usually done by a verification condition generator (VCG), that decomposes the program $m$ according to its syntactic structure.

In a traditional setting without resources, we would use a notion of weakest precondition ( wp $m Q=m \leq$ res $Q$ ), and define rules that syntactically decompose goals of the form $w p m Q$. For example, for sequential composition we have the rule:

$$
w p m(\lambda x . w p(f x) Q) \Longrightarrow w p(x \leftarrow m ; f x) Q
$$

\footnotetext{
${ }^{5}$ This notation was first described in [21, Section 2.2].
} 
In a setting with time, ${ }^{6}$ however, this approach does not work, as the specification $Q$ is not a predicate but a deadline of type $\alpha \rightarrow \gamma$ option that assigns any result a maximum allowed time, or None if that result is not possible.

We solve that problem by generalizing the concept of weakest preconditions from the qualitative to the quantitative domain: instead of only asking whether a program $m$ satisfies a specification res $Q$, we ask how much it satisfies the specification, i. e., what is the latest feasible time at which we can start $m$ to still match the deadline $Q$. We denote this by gwp $m Q:: \gamma$ option (generalized weakest precondition). If the specification is not satisfied, we have gwp $m Q=$ None. In particular, we have the following equalities: $m \leq$ res $Q \Leftrightarrow$ gwp $m Q \neq$ None $\Leftrightarrow$ Some $0 \leq$ gwp $m Q$. Our VCG now operates on goals of the form Some $t \leq \operatorname{gwp} m Q$, and the sequential composition rule reads:

Some $t \leq \operatorname{gwp} m(\lambda x \cdot g w p(f x) Q) \Longrightarrow$ Some $t \leq g w p(x \leftarrow m ; f x) Q$

Formally, we define the generalized weakest precondition as follows:

gwp fail $Q=$ None

gwp (res $M) Q=\operatorname{Inf} r$. minus $(Q r)(M r)$

That is, if the program fails, no starting time is feasible, as expressed by None. Otherwise, we use the most conservative starting time over all possible results, expressed by the infimum (Inf). For a single result, the latest feasible starting time is expressed by the difference of the resources specified and actually used. The difference operator minus $:: \gamma$ option $\rightarrow \gamma$ option $\rightarrow \gamma$ option lifts the difference on resources ${ }^{7}$ to option types. Note that, if the specification cannot be met due to a single result $r$, the difference is None, causing the infimum to be None. Formally, we distinguish the following cases:

- minus (Some $t^{\prime}$ ) (Some $\left.t\right)=$ if $t^{\prime} \geq t$ then Some $\left(t^{\prime}-t\right)$ else None: if the difference is not negative, we return it. Otherwise, the program consumes more resources than specified and does not meet the specification.

- minus None $($ Some $t)=$ None: the result is not covered by the specification, hence the specification cannot be met.

- minus_None = Some $\mathrm{T}$ : the result is not produced by the program, thus it does not contribute to the latest feasible starting time. Accordingly, we return the top element Some T.

It is straightforward to define gwp rules for our monad operations, and construct the desired syntax driven VCG. For details, we refer the reader to [14].

Lockstep Refinement. We often refine a compound program by refining some of its components. For example, in Section 6.3, we replace the specification of the fallback sorting within the abstract introsort algorithm by heapsort.

Let $A$ and $C$ be two structurally equal programs (i.e., they have the same structure of combinators if $f_{c}, \mathrm{rec}_{c}$, bind, etc.), and let $A_{i}$ and $C_{i}$ be the pairs of corresponding basic components, for $i \in\{0, \ldots, n\}$. Provided with refinement lemmas $\left(C_{i}, \lambda x . \Downarrow_{C} E\left(A_{i} x\right)\right) \in\left[\Phi_{i}\right] R_{i} \rightarrow S_{i}$ for each of those pairs, ${ }^{8}$ an automatic procedure walks through the program and establishes a refinement $\left(C, \lambda x . \Downarrow_{C} E(A x)\right) \in[\Phi] R \rightarrow S$. This process generates verification conditions for ensuring the preconditions $\Phi_{i}$, which can be discharged automatically or, if required, via interactive proof.

\footnotetext{
${ }^{6}$ To guide the intuition, we will use time as resource here.

${ }^{7}$ This requires $\gamma$ to provide a difference operator, dual to its + operator. It is a straightforward generalization of the concept defined in [14]. We note that the resource types unit, enat, and ecost provide a suitable difference operator.

${ }^{8}$ The refinement relations $R_{i}$ and $S_{i}$ relate the parameters and respectively the result of those components.
} 
Note that, while the data refinements $R_{i}$ can be different for each component $i$, the exchange rate $E$ must be the same for all components. Currently, we align the exchange rates by manually deriving specialized versions of the component refinement lemmas. While those lemmas are not hard to prove, they are cumbersome to write down. However, we believe that this can be automated in many practical cases, by collecting constraints on the exchange rate during the lockstep refinement, which are solved afterwards to obtain a unified exchange rate. We leave the implementation of this idea to future work.

Separating Analysis of Resource Usage and Correctness. We can disregard resource usage and only focus on refinement of functional correctness, and then add resource usage analysis later. This is useful to separate the concerns of functional correctness and resource usage proof. We will describe a practical example in Section 6.5. Here, we only present an alternative way to prove the refinement from Example 2.7:

First, for functional correctness, we use the specification idxs_cmp $p_{\text {spec }}(\infty)$ and a program $i d x s_{-} c m p_{\infty}$ similar to idxs_cmp but with all the costs replaced by $\infty$. Proving the refinement $i d x s_{c} c m p_{\infty} x s i j \leq i d x s_{-} c m p_{s p e c} x s i j(\infty)$ only requires showing verification conditions that correspond to functional properties and termination, in particular those from assertions and annotated invariants in the concrete program. Proof obligations on resource usage, however, collapse into the trivial $t \leq \infty$. For the same reason, we get $i d x s_{-} c m p x s i j \leq i d x s_{-} c m p_{\infty} x s i j$, and, by transitivity:

$$
i d x s \_c m p x s i j \leq i d x s_{-} c m p \text { spec } x s i j(\infty)
$$

Next, we prove $i d x s_{-}$cmp xs $i j \leq_{n} \operatorname{spec}\left(\lambda_{-}\right.$. True $)\left(\lambda_{-} . \$_{\text {lookup }} 2+\$_{\text {less }}\right)$. Here, the refinement relation $m \leq_{n} m^{\prime}=\left(m \neq\right.$ fail $\left.\Longrightarrow m \leq m^{\prime}\right)$ assumes that the concrete program does not fail. This has the effect that, during the refinement proof, assertions and annotated invariants in the concrete program can be assumed to hold, and we can focus on the resource usage proof.

Finally, the following lemma is used to combine the two refinements:

$$
m \leq \operatorname{spec} P\left(\lambda_{-} . \infty\right) \wedge m \leq_{n} \operatorname{spec}\left(\lambda_{-} . \text {True }\right) T \Longrightarrow m \leq \operatorname{spec} P T
$$

Thus, for our example, we get

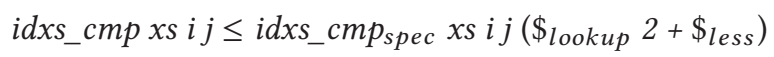

\subsection{Alternatives to NREST}

In the beginning of this section we stated our motivations and design goals for NREST. To model nondeterminism and resources, we used partial functions that map results to resource elements. To motivate this design, we discuss some seemingly obvious alternatives.

A result set and a resource. An alternative would be to define an NREST program being a set of results together with a single resource element for all possible results:

$(\alpha, \gamma) N_{R E S T}=$ fail $\mid$ res $(\alpha$ set $\times \gamma)$

However, this modeling is too coarse: consider a program that modifies a set of natural numbers by repeating the following step until the set is empty: pick and remove a number $n$ from the set, then consume $n$ resources.

Say we start with a set $\{1,2\}$. Then, the result after the first step is res $(\{\{1\},\{2\}\}, 2)$, as there are two possibilities which element was removed from the set, and the upper bound of both outcomes is 2 . After the second step the result must be res $(\{\emptyset\}$, 4), as in both cases the remaining element is removed, but again the upper bound on the running time of that second step is 2 . This yields a total running time of 4 , which is not tight. 
In order to use nondeterminism effectively, we need a finer assignment of resources to results.

A set of pairs. Another alternative is to regard the resource usage just as part of the result. Thus, a set of results with resource usage would be modeled as $(\alpha \times \gamma)$ set. Note that this is isomorphic to $\alpha \rightarrow \gamma$ set, which suits our presentation better. So we define the following alternative to NREST:

$$
(\alpha, \gamma) \operatorname{NREST}_{2}=\text { fail } \mid \text { res }(\alpha \rightarrow \gamma \text { set })
$$

On the one hand, this definition certainly allows to model the two stage process from above adequately. Depending on which number out of $\{1,2\}$ was chosen we can specify a different resource consumption for the intermediate results, and in the end model a tight running time of 3.

On the other hand, the refinement relation cannot just be the natural subset relation, because we would like to have e.g., $\{(x, 3),(x, 4)\} \leq\{(x, 4)\}$, in order to allow refinement with programs with less resource consumption. Formally, we can use a downward closure $(\cdot \downarrow)$ to express refinement:

$$
\begin{aligned}
& S^{\downarrow}=\left\{s \mid \exists s^{\prime} \in S . s \leq s^{\prime}\right\} \\
& \text { res } M \leq \operatorname{res} M^{\prime}=\forall x . M x \subseteq\left(M^{\prime} x\right)^{\downarrow}
\end{aligned}
$$

That is, the computation res $M$ refines res $M^{\prime}$ if for all results $x$ in $M$ the set of possible resource costs is bounded by some possible resource bound for $x$ in $M^{\prime}$.

In our initial design considerations for NREST we dropped that approach because it felt unnatural and the alternative to map results to single resource elements worked out more smoothly. In the following we present some results of a later effort to use the "set of pairs" approach.

First, we note that the refinement defined with the downward closure as above is not antisymmetric, and thus yields no complete lattice structure. This problem, however, can be easily solved by identifying sets with the same downward closure. Technically, we use the quotient type $\gamma$ dclosed $=\gamma$ set $/\left(\lambda s_{1} s_{2} . s_{1} \downarrow=s_{2} \downarrow\right)$, and define a new variant of NREST accordingly:

$$
(\alpha, \gamma) \mathrm{NREST}_{3}=\text { fail } \mid \text { res }(\alpha \rightarrow \gamma \text { dclosed })
$$

For this, we straightforwardly get the desired complete lattice structure on $\mathrm{NREST}_{3}$. We even get a more elegant formalization, as the empty set $\left(\emptyset^{\downarrow}\right)$ naturally models the case where no result is present, and the universal set $\left(U N I V^{\downarrow}\right)$ is the greatest element. In our original NREST, we had to use partial functions to model absence of results, and add artificial greatest elements to the resource type (e.g., $\infty$ in enat).

For a resource type that provides a neutral element 0 and addition + with a monoid structure, we further can define the monadic operators return, bind and elapse as expected. The lifting of + to downward closed sets, as required for defining bind, is straightforward.

However, we got stuck when we tried to define generalized weakest preconditions (cf. Section 2.6) in $\mathrm{NREST}_{3}$, more precisely, the underlying difference operator on resources. For example, consider the following scenario where resources have more than one extreme point: we assume resources with two currencies, expressed as pairs of amounts. Let $\{(2,0),(0,2)\}^{\downarrow}$ be the specified resources for some result and $\{(1,0),(0,1)\}^{\downarrow}$ the ones actually required by the program. In order to determine gwp, we would have to take the difference of these two downward closed sets. However, it is unclear to us how to define the difference in a sensible way.

In our actual NREST design, however, we aggregate the cost into one element. We would obtain $(2,2)$ and $(1,1)$, respectively, and the difference operator can easily be defined pointwise. We have to note that the overapproximation of $\{(2,0),(0,2)\}$ to $(2,2)$ does cause a problem, which we will treat in Section 4.5.

In summary, our choice of modeling NREST by one resource element per possible result seems to be a sweet spot: it is fine enough to model nondeterminism effectively and coarse enough to define generalized weakest preconditions. 


\section{LLVM WITH COST SEMANTICS}

The NREST-monad allows to specify programs with their resource usage in abstract currencies. Those currencies only have a meaning when they finally can be exchanged for the costs of concrete computations. In the following, we present such a concrete computation model, namely a shallow embedding of the LLVM semantics into Isabelle/HOL. The embedding is an extension of our earlier work [22] to also account for costs. In Section 4, we will then report on linking the LLVM back end with the NREST front end.

\subsection{Basic Monad}

At the basis of our LLVM formalization is a monad that provides the notions of non-termination, failure, state, and execution costs.

$$
\begin{aligned}
& \alpha \text { mres }=\text { NTERM } \mid \text { FAIL } \mid \text { SUCC } \alpha \text { cost state } \\
& \alpha M=\text { state } \rightarrow \alpha \text { mres }
\end{aligned}
$$

Here, cost is a type for execution costs, which forms a monoid with operation + and neutral element 0 , and state is an arbitrary type. ${ }^{9}$

The type $\alpha M$ describes a program that, when executed on a state, either does not terminate (NTERM), fails (FAIL), or returns a result of type $\alpha$, its execution costs, and a new state (SUCC).

It is straightforward to define the monad operations return and bind, as well as a recursion combinator rec over $M$. Thanks to the shallow embedding, we can also use Isabelle HOL's if-thenelse to get a complete set of basic operations. As an example, we show the definition of the bind operation, in the case that both arguments successfully compute a result:

Assume $m s=\operatorname{SUCC} x c_{1} s_{1}$ and $f x s_{1}=\operatorname{SUCC} r c_{2} s_{2}$

then we have bind $m f s=S U C C r\left(c_{1}+c_{2}\right) s_{2}$

That is, the result $x$ and state $s_{1}$ after the first operation $m$ is passed into the second operation $f$, and the result and state after the bind is what emerges from $f$. The cost for the bind is the sum of the costs for both operations.

The basic monad operations do not cost anything. To account for execution costs, we define an explicit operation consume $c s=\operatorname{SUCC}() c s .{ }^{10}$

\subsection{Shallowly Embedded LLVM Semantics}

The formalization of the LLVM semantics is organized in layers. At the bottom, there is a memory model that stores deeply embedded values, and comes with basic operations for allocation/deallocation, loading, storing, and pointer manipulation. Also the basic arithmetic operations are defined on deeply embedded integers. These operations are phrased in the basic monad, but consume no costs. This way, we could take them unchanged from our original LLVM formalization without cost [22]. For example, the low-level load operation has the signature raw_load : raw_ptr $\rightarrow$ val M. Here, raw_ptr is the pointer type of our memory model, consisting of a block address and an offset, and val is our value type, which can be an integer, a pointer, or a pair of values.

On top of the basic layer, we define operations corresponding to the actual LLVM instructions. Here, we map from deeply to shallowly embedded values, and add the execution costs.

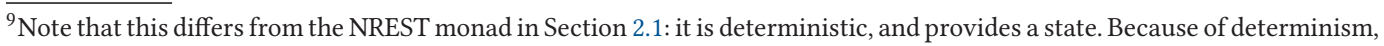
we never need to form a supremum, and thus can base our cost model on natural numbers rather than enats. We leave a unification of the two monads to future work.

${ }^{10}$ For NREST, we defined a higher-order operation elapse, while we use the first-order operation consume here. This is for historical reasons. Note that elapse can be defined in terms of consume, and vice versa.
} 
For example, the semantics of LLVM's load instruction is defined as follows:

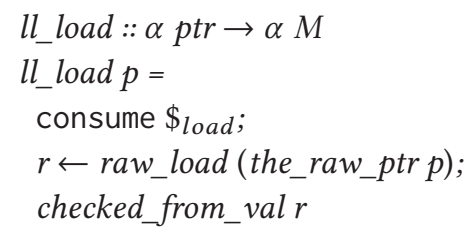

It consumes the cost ${ }^{11}$ for the operation, and then forwards to the raw_load operation of the lower layer, where the_raw_ptrand checked_from_val convert between the shallow and deep embedding of values.

Like in the original formalization, ${ }^{12}$ an LLVM program is represented by a set of monomorphic constant definitions of the shape def, defined as follows:

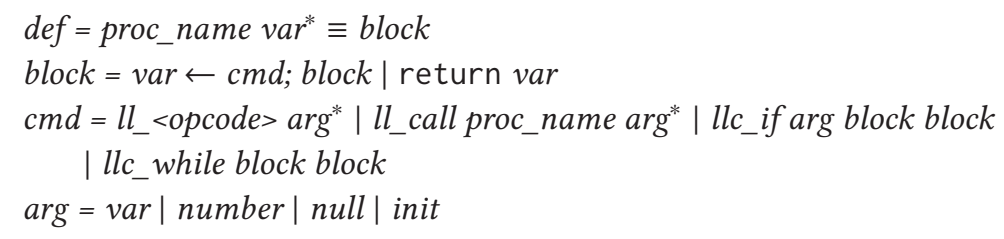

The code generator checks that the set of definitions is complete and adheres to the required shape. It then translates them into LLVM code, which merely amounts to pretty printing and translating the structured control flow by if and whil $\mathrm{e}^{13}$ statements to the unstructured control flow of LLVM. A powerful preprocessor can convert a more general class of terms to the restricted shape required by the code generator. This conversion is done inside the logic, i.e., the processed program is proved to be equal to the original. Preprocessing steps include monomorphization of polymorphic constants, extraction of fixed-point combinators to recursive function definitions, and conversion of tuple constructors and destructors to LLVM's insertvalue and extractvalue instructions.

In summary, the layered architecture of our LLVM formalization allowed for a smooth integration of the cost aspect, reusing most of the existing formalization nearly unchanged. Note that we opted to integrate the cost aspect into the existing top layer, which converts between deep and shallow embedding. Alternatively, we could have added another layer on top of the shallow embedding. While the latter would have been the cleaner design, we opted for the former approach to avoid the boilerplate of adding a new layer. This was feasible as the original top layer was quite thin, such that adding another aspect there did not result in excessive complexity.

\subsection{Cost Model}

As a cost model for running time, we chose to count how often each instruction is executed. That is, we set cost $=$ string $\rightarrow$ nat, where the string encodes the name of an instruction. It is straightforward to define 0 and + such that (cost, $0,+)$ forms a monoid. It is thus a valid cost model for our monad.

But how realistic is our cost model, counting LLVM instructions? During compilation, LLVM text will be transformed by LLVM's optimizer, and finally, the LLVM back end will translate LLVM

\footnotetext{
${ }^{11}$ See Section 3.3 for an explanation of our cost model.

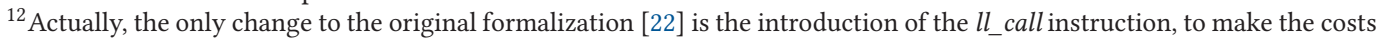
of a function call visible.

${ }^{13}$ Primitive while loops are not strictly required, as they can always be replaced by tail recursion. Indeed, our code generator can be configured to not accept while loops, and our preprocessor can automatically convert while loops to tail-recursive functions. However, the efficiency of the generated code then relies on LLVM's optimization pass to detect the tail recursion and transform it to a loop again.
} 
instructions to machine instructions. Moreover, the actual running time of a machine program does not only depend on the number of executed instructions, but effects like pipeline flushes and cache misses also play an important role. Thus, without factoring in the details of the optimization passes and the target machine architecture, our cost model can, at best, be a rough approximation of the actual running time.

However, we do assume that a single instruction in the original LLVM text will result in at most a (small) constant number of machine instructions, and that each machine instruction has a constant worst-case execution time. Thus, the steps counted by our model linearly correlate to an upper bound of the actual execution time, though the exact correlation depends on the actual program, optimizer passes, and target architecture. Hence, while our cost model cannot be used for precise statements about execution time, it can be used to prove worst-case complexity. That is, a program that we have proved efficient will be compiled to an efficient machine program. Moreover, we can hope that the constant factors in the proved complexity are related to the actual constant factors in the machine program, i.e., an LLVM program with small constant factors will compile to a machine program with small constant factors.

The above discussion justifies the following design choices: The insertvalue and extractvalue instructions, which are used to construct and destruct tuple values, have no associated costs. The main reason for this design is to enable transparent use of tupled values, e.g., to encode the state of a while loop. We expect LLVM to translate the members of the tuple to separate registers anyway, such that no real costs are associated with tupling/untupling.

We define the malloc instruction to take cost proportional to the number of allocated elements. ${ }^{14}$ Note that LLVM itself does not provide memory management, and our code generator forwards memory management instructions to the libc implementation of the target platform. We use the calloc function here, which is supposed to initialize the allocated memory with zeros. While the exact costs of that are implementation dependent, they certainly will depend on the size of the allocated block.

Charguéraud and Pottier [7, Section 2.7] discuss the adequacy of abstract cost models in a functional setting. In their classification, our abstraction would be on Level 2, as we count (almost) all kinds of operations on an intermediate language level.

\subsection{Reasoning Setup}

Once we have defined the semantics, we need to set up some basic reasoning infrastructure. The original Isabelle-LLVM already comes with a quite generic separation logic and verification condition generation framework. Here, we report on our extensions to resources using time credits.

Separation Logic with Time Credits. Our reasoning infrastructure is based on separation logic with time credits $[1,7,13]$. We follow the algebraic approach of Calcagno et al. [3], using an earlier extension [22] of Klein et al. [25].

A separation algebra on type $\alpha$ induces a separation logic on assertions that are predicates over $\alpha$. To guide intuition, elements of $\alpha$ are called heaps here. We use the following separation logic operators: The assertion $\uparrow \Phi$ holds for an empty heap if $\Phi$ holds, $\square=\uparrow$ True describes the empty heap, and $\exists_{A}$ is the existential quantifier lifted to assertions. The separating conjunction $P \star Q$ describes a heap comprised from two disjoint parts, one described by $P$ and the other described by $Q$, and entailment $P \vdash Q$ states that $Q$ holds for every heap described by $P$.

Separation algebras naturally extend over product and function types, i.e., for separation algebras $\alpha, \beta$, and any type $\gamma$, also $\alpha \times \beta$ and $\gamma \rightarrow \alpha$ are separation algebras, where the operations are lifted pointwise.

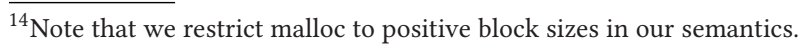


Note that enat forms a separation algebra, where elements, i.e., time credits, are always disjoint. Hence, also ecost $=$ string $\rightarrow$ enat, and amemory $\times$ ecost are separation algebras, where amemory is the separation algebra that we already used in [22] to describe the abstract memory of LLVM. Thus, amemory $\times$ ecost induces a separation logic with time credits that match our cost model. The time credit assertion $\$ t=(\lambda a . a=(0, t))$ describes an empty memory $(0)$ and precisely the time $t^{15}$ The primitive assertions on amemory are lifted analogously to describe no time credits.

Weakest Precondition and Hoare Triples. We start by defining a concrete state cstate that describes the memory content and the available resources:

cstate $=$ memory $\times$ ecost

where memory is the memory type from our original LLVM formalization. Based on this, we define the weakest precondition predicate:

$$
\begin{aligned}
& w p:: \alpha M \rightarrow(\alpha \rightarrow \text { cstate } \rightarrow \text { bool }) \rightarrow \text { cstate } \rightarrow \text { bool } \\
& \text { wp } m Q(s, c c)=\left(\exists r c s^{\prime} . m s=S U C C r c s^{\prime} \wedge c \leq c c \wedge Q r\left(s^{\prime}, c c-c\right)\right) .
\end{aligned}
$$

Intuitively, the costs $c c$ stored in the state is the credit available to the program. The weakest precondition holds if the program runs with real costs $c$ that are within the available credit, and $Q$ holds for the result $r$, the new memory $s^{\prime}$, and the new credit, $c c-c$, which is the old credit reduced by the actually required costs. Note that actual costs have type cost $=$ string $\rightarrow$ nat, i.e., are always finite, while the credits have type ecost $=$ string $\rightarrow$ enat, i.e., there can be infinite credits. Setting the credit to be infinite for all instruction types yields the classical weakest precondition that requires termination, but enforces no time limit.

Our concrete state type, in particular the memory, does not form a separation algebra, as the natural memory model of LLVM has no notion of partial memories. Thus, we define an abstraction function that maps a concrete state to an abstract state astate, which forms a separation algebra:

$$
\text { astate }=\text { amemory } \times \text { ecost } \quad a b s(m, c)=\left(a b s_{m} m, c\right)
$$

Again, amemory and $a b s_{m}$ are the abstract state and abstraction function from the original LLVM formalization. The costs already form a separation algebra, so we do not abstract them further.

With this, we can instantiate a generic VCG infrastructure: let cstate be the type of concrete states, wp :: $\alpha M \rightarrow(\alpha \rightarrow$ cstate $\rightarrow$ bool $) \rightarrow$ cstate $\rightarrow$ bool be a weakest precondition predicate, and astate the type of abstract states, linked to concrete states via an abstraction function $a b s::$ cstate $\rightarrow$ astate. In order to weaken postconditions, we assume that $w p$ is monotone, i.e.,

$$
\left(\forall x . Q x \Longrightarrow Q^{\prime} x\right) \Longrightarrow w p c Q s \Longrightarrow w p c Q^{\prime} s
$$

Finally, let $\pi$ be an affine top [5], i.e., an assertion with $\square \vdash \pi$ and $\pi \star \pi=\pi$, which captures resources that can be safely discarded. We define the Hoare triple $\{P\} c\{Q\}$ to hold iff:

$$
\forall F s .(P \star F)(a b s s) \Longrightarrow w p c\left(\lambda r s^{\prime} .(Q r \star \pi \star F)\left(a b s s^{\prime}\right)\right) s
$$

Intuitively, $\{P\} c\{Q\}$ holds if, for all states that contain a part described by assertion $P$, command $c$ terminates with result $r$ and a state where that part is replaced by a part described by $Q r \star \pi$, and the rest of the state has not changed. Here, $Q r$ is the postcondition of the Hoare triple, and $\pi$ describes resources that may be left over and can be discarded.

In our case, we set $\pi$ to describe the empty memory and any amount of time credits. This matches the intuition that a program must free all its memory, but may run faster than estimated, i.e., leave over some time credits. Note that our $w p$ is monotone.

\footnotetext{
${ }^{15}$ Beware of the notation $\$ \$ c$, which asserts one coin of the currency $c$.
} 
The generic VCG infrastructure now provides us with a syntax driven VCG with a simple frame inference heuristics.

\subsection{Primitive Setup}

Once we have defined the basic reasoning infrastructure, we have to prove Hoare triples for the basic LLVM instructions and control flow combinators. As we have added the cost aspect only at the top level of our semantics, we can reuse most of the material from our original LLVM formalization without time. Technically, we instantiate our reasoning infrastructure with a weakest precondition predicate $w p n$, which only holds for programs that consume no costs. We define:

wpn $m Q s=$ wp $m(F S T \circ Q)(s, 0)$ where FST $P=\lambda(s, c) . P s \wedge c=0$

Here, FST lifts an assertion on the first component to an assertion on a pair.

The resulting reasoning infrastructure is identical with the one of our original formalization, most of which could be reused. Only for the topmost level, i.e., for those functions that correspond to the functional semantics of the actual LLVM instructions, we lift the Hoare triples over wpn to Hoare triples over $w p$ :

$$
\{P\} c\{Q\}_{w p n}=\{F S T P\} c\{F S T \circ Q\}
$$

Example 3.1. Recall the low-level raw_load and the high-level ll_load instruction from Section 3.2. The raw_load instruction consumes no costs, and our original LLVM formalization provides the following Hoare triple:

$$
\left\{r a w_{-} p t o p x\right\} \text { raw_load } p\left\{\lambda r . \uparrow(r=x) \star r a w_{-} p t o p x\right\}_{w p n}
$$

This can be transferred to a Hoare triple over $w p$ :

$$
\{F S T(\text { raw_pto } p x)\} \text { raw_load } p\{\lambda r . \uparrow(r=x) \star F S T(\text { raw_pto } p x)\}
$$

which is then used to prove the Hoare triple for the program $l l_{-}$load

$$
\left\{\text { pto } p x \star \$ \$_{\text {load }}\right\} \text { ll_load } p\{\lambda r . \uparrow(r=x) \star p t o p x\}
$$

where pto $p x=$ FST (raw_pto (the_raw_ptrp) (to_val $x)$ ).

Using the VCG and the Hoare triples for the LLVM instructions, we can now define and prove correct data structures and algorithms. While this works smoothly for simple data structures like arrays, it does not scale to more complex developments. In contrast, NREST does scale, but lacks support for the low-level pointer reasoning required for basic data structures. In Section 4, we show how to combine both approaches, with the LLVM level providing basic data structures and the NREST level using them as building blocks for larger algorithms.

\subsection{Free for Free}

Note that in our semantics, both memory allocation and memory deallocation consume costs of currencies malloc and free, respectively. However, the automatic data refinement tool we are going to design (see Section 4.2) has to automatically insert destructors, which free memory. A destructor $d$ that destroys an object described by assertion $A$ is characterized in the following way:

$$
\text { destructor } A d=(\forall a c .\{A a c\} d c\{\square\})
$$

In particular, all costs required for destruction must already be contained in the assertion $A$. In practice, this means that we pay for the destruction of an object upon its allocation. Thus, we prove the following Hoare triples for allocation and deallocation: 


$$
\begin{aligned}
& \{\$(\$ \text { malloc } n+\$ \text { free }) \star \uparrow(n>0)\} \\
& \quad l l \_ \text {malloc } \alpha n \\
& \left\{\lambda p \text {.range }\{0 . .<n\}\left(\lambda \_. \text {init }\right) p \star \text { malloc_tag } n p\right\} \\
& \{\text { range }\{0 . .<n\} \text { blk } \star \text { malloc_tag } n p\} \text { ll_free } p\{\square\}
\end{aligned}
$$

Intuitively, to allocate a block of size $n$, one has to pay $n$ units of malloc and 1 unit of free. To free a block, no explicit costs have to be paid.

Note that the malloc_tag assertion in the original formalization expresses ownership on the whole block and is a prerequisite for freeing a block. Thus, it was natural to add the required time credits for freeing to this assertion, when extending the original formalization with time:

$$
\text { malloc_tag } n p=F S T\left(r a w_{-} \text {malloc_tag } n\left(t h e \_r a w \_p t r p\right)\right) \star \$ \$ \text { free }
$$

where raw_malloc_tag is the ownership assertion from our low-level memory model.

Note how amortization arguments like the above are seamlessly supported by separation logic with time credits [1]. Later in this article (Section 5) we also show how to combine amortization with refinement.

In practice, the malloc_tag assertion is usually hidden in the assertion for a data structure, and thus not directly visible to the user.

\subsection{Modeling Data Structures}

An imperative data structure is described by a refinement assertion that relates it to a functional model. The refinement assertion usually contains the addresses and block ownership (malloc_tag) for all memory used to represent the data structure. For each operation, a Hoare triple is proved that relates the concrete operation on the heap to the corresponding abstract operation on the functional model.

For example, the assertion $\operatorname{array}_{A} x s p$ relates the array pointed to by $p$ to the list $x s$ of its elements:

$$
\operatorname{array}_{A} x s p=\text { range }\{0 . .<|x s|\}(\lambda i . x s ! i) p \star \text { malloc_tag }|x s| p
$$

Note that we sometimes use the suffix ${ }_{A}$ to make clear that a name refers to an assertion.

The following Hoare triples relate the standard array operations to the corresponding operations on lists:

$$
\begin{aligned}
& \left\{\$\left(\$_{\text {malloc }} n+\$_{\text {free }}\right) \star \uparrow(n>0)\right\} \text { array_new } \alpha n\left\{\lambda p \text {. } \operatorname{array}_{A}(\text { replicate } n \text { init }) p\right\} \\
& \left\{\text { array }_{A} x s p\right\} \text { array_delete } p\{\square\} \\
& \left\{\text { array }_{A} x s p \star \$\left(\$_{\text {ofs_ptr }}+\$ \$_{\text {load }}\right) \star \uparrow(i<|x s|)\right\} \text { array_get } p i\left\{\lambda r \text {. } \text { array }_{A} x s p \star \uparrow(r=x s ! i)\right\} \\
& \left\{\text { array }_{A} x s p \star \$\left(\$_{\text {ofs_ptr }}+\$_{\text {store }}\right) \star \uparrow(i<|x s|)\right\} \text { array_set }_{-} \text {ix }\left\{\lambda \lambda_{\text {. }} \operatorname{array}_{A}(x s[i:=x]) r\right\}
\end{aligned}
$$

Users of the array data structure only need to use this interface, and never have to look into the details of the implementations or the refinement assertion.

Note that, as described in Section 3.6, we pay the cost for destruction already upon construction. For a simple array, the destructor only invokes $l l$ free, whose costs are already contained in malloc_tag. More complicated data structures, however, may require additional costs for destruction (e.g., to traverse a list of allocated arrays). These can also be hidden in the refinement assertion. 


\section{AUTOMATIC REFINEMENT}

In this section, we describe a tool to synthesize a concrete program in the LLVM-monad from an abstract algorithm in the NREST-monad. It can automatically refine abstract functional data structures to imperative heap-based ones. We will describe the synthesis predicate $h n r$ that connects the two monads, the synthesis tool, and a way to extract Hoare triples from $h n r$ predicates. Finally, we will discuss an effect that prevents combining $h n r$ with data refinements in the NREST-monad in the general case.

\subsection{Heap Nondeterminism Refinement}

The heap nondeterminism refinement predicate $h n r \Gamma m_{\dagger} \Gamma^{\prime} A m$ intuitively expresses that the concrete program $m_{\dagger}$ computes a concrete result that relates, via the refinement assertion $A$, to a result in the abstract program $m$, using at most the resources specified by $m$ for that result. A refinement assertion describes how an abstract variable is refined by a concrete value on the heap. It can also contain time credits. The assertions $\Gamma$ and $\Gamma^{\prime}$ constitute the heaps before and after the computation and typically are a separating conjunction of refinement assertions for the respective parameters of $m_{\dagger}$ and $m$. Formally, we define:

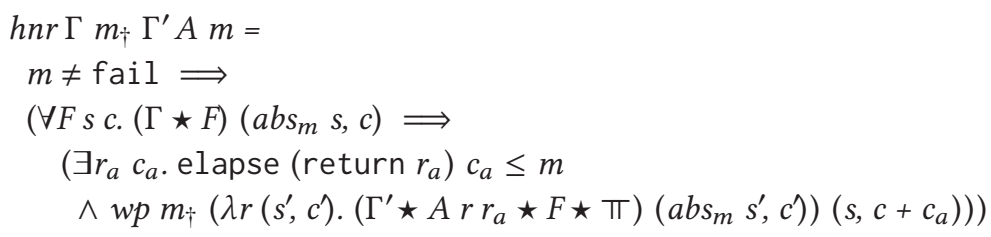

The predicate holds if either the abstract program fails or if, for all heaps and resources $(s, c)$ that satisfy the pre-assertion $\Gamma$ with some frame $F$, there exists an abstract result and cost $\left(r_{a}, c_{a}\right)$ that refine $m$, and $m_{\dagger}$ terminates with concrete result $r$ in a state $s^{\prime}$ where $\Gamma^{\prime}$ with the frame holds, and $r$ relates to the abstract result via assertion $A$. The execution costs of $m_{\dagger}$ and the time credits $c^{\prime}$ required by the post-assertion $\Gamma^{\prime}$ are paid for by the specified cost $c_{a}$ and the time credits $c$ described by the pre-assertion $\Gamma$. Thus, the real costs are paid by a combination of the advertised costs in the abstract program and the potential difference of $\Gamma^{\prime}$ and $\Gamma$, allowing to seamlessly model amortized computation costs.

The affine top $\pi$ allows the program to throw away portions of the heap. Note that our $\pi$ can only discard time credits. Memory must be explicitly freed by the concrete program $m_{\dagger}$.

Also note that $h n r$ is not tied to the LLVM semantics specifically. It actually is a general pattern for combining the NREST-monad with any other program semantics that provides a weakest precondition and a separation algebra for data and resources.

\subsection{The Sepref Tool}

The Sepref tool $[20,22]$ automatically synthesizes a concrete program in the LLVM-monad from an abstract algorithm in the NREST-monad. It symbolically executes the abstract program while maintaining refinements for the abstract variables to a concrete representation and generates a concrete program as well as a valid $h n r$ predicate. Proof obligations ${ }^{16}$ that occur during this process are discharged automatically, guided by user-provided hints where necessary.

The synthesis requires rules for all abstract combinators. For example, bind is processed by the following rule:

$1 \quad\left(h n r \Gamma m_{\dagger} \Gamma^{\prime} A_{x} m \wedge\right.$

$2 \quad\left(\forall x x_{\dagger} \cdot h \operatorname{hnr}\left(A_{x} x_{\dagger} x \star \Gamma^{\prime}\right)\left(f_{\dagger} x_{\dagger}\right)\left(A_{x}^{\prime} x_{\dagger} x \star \Gamma^{\prime \prime}\right) A_{y}(f x)\right) \wedge$

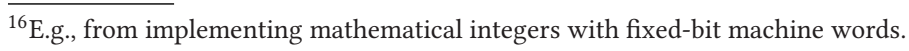




$$
\begin{aligned}
& 3 \\
& \left.4 \quad \text { destructor } A_{x}^{\prime} \text { free }\right) \Longrightarrow \\
& \Rightarrow n r \Gamma\left(x_{\dagger} \leftarrow m_{\dagger} ; r_{\dagger} \leftarrow f_{\dagger} x_{\dagger} ; \text { free } x_{\dagger} \text {; return } r_{\dagger}\right) \Gamma^{\prime \prime} A_{y}(x \leftarrow m ; f x)
\end{aligned}
$$

To refine $x \leftarrow m$; $f x$, we first execute $m$, synthesizing the concrete program $m_{\dagger}$ (line 1 ). The state after $m$ is $A_{x} x_{\dagger} x \star \Gamma^{\prime}$, where $x$ is the result created by $m$. From this state, we execute $f x$ and synthesize $f_{\dagger} x_{\dagger}$ (line 2). The new state is $A_{x}^{\prime} x_{\dagger} x \star \Gamma^{\prime \prime} \star A_{y} y_{\dagger} y$, where $y$ is the result of $f x$. Now, the intermediate variable $x$ goes out of scope and has to be deallocated. The predicate destructor $A_{x}^{\prime}$ free (line 3) states that free is a deallocator for data structures implemented by refinement assertion $A_{x}^{\prime}$. Note that free can only use time credits that are stored in $A_{x}^{\prime}$. Typically, these are payed for during creation of the data structure (cf. Section 3.6). This way amortization can be used effectively to hide the necessary free operation and its costs in the abstract program.

All other combinators $\left(\mathrm{rec}_{c}\right.$, if $\mathrm{f}_{c}$, while $\mathrm{e}_{c}$, etc.) have similar rules that are used to decompose an abstract program into parts, synthesize corresponding concrete parts recursively and combine them afterwards with the respective combinators from LLVM. At the leaves of this decomposition, atomic operations need to be provided with suitable synthesis predicates.

An example is a list lookup that is implemented by an array:

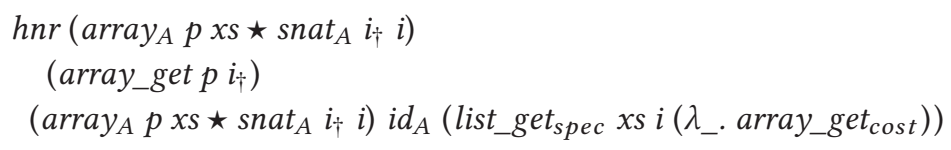

Here, the assertions $\operatorname{array}_{A}$, $s n a t_{A}$, and $i d_{A}$ relate a list with an array, an unbounded natural number with a bounded signed word and identical elements, respectively. With an array at address $p$ holding the list xs and an index $i_{\uparrow}$ that is a bounded signed word representing an unbounded natural number $i$, array_get leaves the parameters unchanged and extracts the element specified by list_get $t_{\text {spec }}$ incurring costs array_get $t_{\text {cost }}=\$_{\text {ofs_ptr }}+\$_{\text {load }}$.

Ideally, each operation has its own currency (e.g., list_get). However, as our definition of $h n r$ does not support currency refinement, the basic operations must use the currencies of the LLVM cost model. To still obtain modular $h n r$ rules, we encapsulate specifications for data structures with their cost, e.g., by defining array_get $t_{s p e c}=$ list_get $_{\text {spec }}\left(\lambda_{-}\right.$. array_get $\left.t_{\text {cost }}\right)$. These can easily be introduced in an additional refinement step. Automating this process, and possibly integrating currency refinement into $h n r$ is left to future work.

\subsection{Notation for Refinement}

Synthesis rules typically have the following general form:

$$
\begin{aligned}
& P\left(x_{1 \dagger}, \ldots, x_{n \dagger}\right)\left(x_{1}, \ldots, x_{n}\right) \Longrightarrow \\
& h n r\left(A_{1} x_{1 \dagger} x_{1} \star \ldots \star A_{n} x_{n \dagger} x_{n}\right) \quad\left(f_{\dagger}\left(x_{1 \dagger}, \ldots, x_{n \dagger}\right)\right) \\
& \left(A_{1}^{\prime} x_{1 \dagger} x_{1} \star \ldots \star A_{n}^{\prime} x_{n} \dagger x_{n}\right) A\left(f\left(x_{1}, \ldots, x_{n}\right)\right)
\end{aligned}
$$

That is, if we have concrete parameters $x_{1 \dagger}, \ldots, x_{n \dagger}$ that refine the abstract parameters $x_{1}, \ldots, x_{n}$, wrt. refinement assertions $A_{1}, \ldots, A_{n}$, and, additionally, the precondition $P$ holds for the parameters, then the result of the concrete function $f_{\dagger}$ applied to the concrete parameters refines the result of the abstract function applied to the abstract parameters, with assertion $A$. Moreover, after executing the function, some parameters $x_{i \dagger}$ may still be valid, e.g., if they are only read. In this case, we have $A_{i}{ }^{\prime}=A_{i}$. For parameters that are deleted by the function, or whose ownership is transferred (e.g. into the result), we have $A_{i}{ }^{\prime}=\operatorname{del} A_{i} \cdot{ }^{17}$

\footnotetext{
${\overline{{ }^{17} \text { Here, } \operatorname{del} A x_{\dagger}}}_{x}=\uparrow\left(\exists h . A x_{\dagger} x h\right)$ just retains the information that the assertion is true for some heap (e.g. the original one). Our framework uses this information to restore the parameter in case the refinement assertion is pure, i.e., does not depend on the heap.
} 
We introduce a more succinct notation for synthesis rules of the above form: ${ }^{18}$

$\left(f_{\dagger}, f\right) \in[P] A_{1} p_{1} \rightarrow \ldots \rightarrow A_{n}^{p_{n}} \rightarrow A$

The notation is inspired by relational parametricity rules. The superscripts of the refinement assertions indicate whether the parameter will be kept on the heap $\left(A_{i}{ }^{\prime}=A_{i}\right)$ or destroyed $\left(A_{i}{ }^{\prime}=\operatorname{del} A_{i}\right)$.

Example 4.1. Given assertions $L_{A}$ and $E_{A}$, the following expresses the correctness of an implementation push $h_{\dagger}$ of list_push $_{\text {spec }}$ :

$$
\left(\text { push }_{\dagger}, \text { list_push }_{\text {spec }}(t)\right) \in L_{A}{ }^{d} \rightarrow E_{A}{ }^{k} \rightarrow L_{A}
$$

That is, the first parameter (the list) is refined by the assertion $L_{A}$. The ${ }^{d}$ annotation expresses that our implementation destructively updates the list, i.e., ownership of the list is transferred into the result. The second parameter (the element) is refined by the assertion $E_{A}$. The ${ }^{k}$ annotation expresses that our implementation does not change the parameter. ${ }^{19}$ Finally, the result list is, again, refined by the assertion $L_{A}$.

In Section 5, we will provide such an implementation with dynamic arrays.

\subsection{Extracting Hoare Triples}

Note that $h n r$ predicates cannot always be expressed as Hoare triples, as the running time bound of the abstract program may depend on the result, which we cannot refer to in the precondition of a Hoare triple, where we have to express the allowed running time as time credits. ${ }^{20}$

$$
h n r \Gamma m_{\dagger} \Gamma^{\prime} A\left(\operatorname{spec} \Phi\left(\lambda_{-} . t\right)\right)=\{\$ t \star \Gamma\} m_{\dagger}\left\{\lambda r . \Gamma^{\prime} \star \exists_{A} r_{a} . A r r_{a} \star \uparrow\left(\Phi r_{a}\right)\right\}
$$

While intermediate components might not be of this form, final algorithms typically are. At the end of a development, this rule allows to extract a Hoare triple in the underlying LLVM semantics, cutting out the NREST-monad. For validating the correctness claim of an algorithm, only the final Hoare triple needs to be inspected, which only uses concepts of the underlying semantics.

Note that the above rule is an equivalence. Thus, it can also be used to obtain synthesis rules from Hoare triples provided by the basic VCG infrastructure.

\subsection{Attain Supremum}

We comment on a problem that arises when composing hnr predicates and data refinement in the NREST monad. Consider the following programs and relations:

$$
\begin{array}{ll}
m^{\prime}=\operatorname{res}\left[x \mapsto \$_{a}, y \mapsto \$_{b}\right] & \mathrm{R}=\{(\mathrm{z}, \mathrm{x}),(\mathrm{z}, \mathrm{y})\} \\
m=\operatorname{res}\left[z \mapsto \$_{a}+\$_{b}\right] & \mathrm{A}=\mathrm{id}_{A} \\
m_{\dagger}=\text { consume }\left(\$_{a}+\$_{b}\right) \text {; return } z &
\end{array}
$$

The specification $m^{\prime}$ returns the abstract result $x$ at cost $\$_{a}$ or $y$ at cost $\$_{b}$. The program $m$ returns the concrete result $z$ at cost $\$_{a}+\$_{b}$. The LLVM program $m_{\dagger}$ also returns $z$ at cost $\$_{a}+\$_{b}$. The relation $R$ relates $z$ with both, $x$ and $y$. The assertion $A$ relates identical elements.

Data refinement defines the resource bound for a concrete result (here $z$ ) as the supremum over all bounds of related results (here $x, y$ ). Thus, we have $m \leq$ res $\left[z \mapsto \$_{a}+\$_{b}\right]=\Downarrow_{D} R m^{\prime}$. Moreover, we trivially have $h n r \square m_{\dagger} \square A m$. Intuitively, we want to compose these two refinements, to obtain

\footnotetext{
${ }^{18}$ The notation is introduced by Lammich e. g., in [23, Section 5.1].

${ }^{19}$ Note that this requires the implementation to copy the element into the array rather than to just transfer its ownership. ${ }^{20}$ Guéneau et al. $[11,13]$ resolve that limitation by the introduction of possibly-negative time credits. However, the crucial equivalence of positive credits in the precondition with negative credits in the postcondition does not hold when allowing infinite credits. As infinite credits are important for our approach, and the low-level definition of hnr is viable (though less aesthetic), we did not pursue this further.
} 
$h n r \square m_{\dagger} \square(A \circ R) m^{\prime}$. However, as our definition of $h n r$ does not form a supremum, this would require $\$_{a}+\$_{b} \leq \$_{a}$ or $\$_{a}+\$_{b} \leq \$_{b}$, which obviously does not hold.

We have not yet found a way to define $h n r$ or $\Downarrow_{D}$ in a form that does not exhibit this effect. Instead, we explicitly require that the supremum of the data refinement has a witness. The predicate attains_sup $m m^{\prime} R$ characterizes that situation: it holds, if for all results $r$ of $m$ the supremum of the set of all abstractions $\left(r, r^{\prime}\right) \in R$ applied to $m^{\prime}$ is in that set. This trivially holds if $R$ is single-valued, i.e., any concrete value is related with at most one abstract value, or if $m^{\prime}$ is one-time, i.e., assigns the same resource bound to all its results.

In practice we $d o$ encounter non-single-valued relations, ${ }^{21}$ but they only occur as intermediate results where the composition with an hnr predicate is not necessary. Also, collapsing synthesis predicates and refinements in the NREST-monad typically is performed for the final algorithm whose running time does not depend on the result, thus is one-time, and ultimately attains_sup.

\section{CASE STUDY: DYNAMIC ARRAYS IN THE ABSTRACT}

In this section, we present a case study that shows that amortized data structures can be proven correct on the abstract NREST level. We verify the amortized-constant-time push operation of dynamic arrays in the abstract NREST formalism and then synthesize LLVM code from it using the automatic method from the previous section. We focus on the resource consumption and the amortization argument in particular. For presentation purposes we omit functional correctness and some size side conditions that are vital for the implementation in LLVM. We will comment on that towards the end of this section.

\subsection{Dynamic Lists}

In Example 2.6, we introduced dynamic lists, which model dynamic arrays as a triple of a carrier list, its length, and its capacity. We have shown that $d l_{-} p u s h_{\text {spec }}$ on dynamic lists refines list_push $h_{\text {spec }}$ on lists (Example 2.9). The next step in refining the push operation is to add the abstract algorithmic idea: If we run out of capacity, we double the size of the carrier list and push the element afterwards.

$$
\begin{aligned}
& d l \_p u s h(c s, l, c) x= \\
& \text { if } \mathrm{f}_{c} l<c \text { then } \quad \text { (\$less) } \\
& d l_{-} p u s h \_b a s i c_{s p e c}(c s, l, c) x \quad\left(\$ d l_{-} \text {push_basic }\right) \\
& \text { else } \\
& \left(c s^{\prime}, l^{\prime}, c^{\prime}\right) \leftarrow d l_{-} \text {double } \text { spec }(c s, l, c) ; \quad\left(\$_{d l \_d o u b l e_{c}} \mathrm{c}\right) \\
& d l \_p u s h \_b a s i c_{s p e c}\left(c s^{\prime}, l^{\prime}, c^{\prime}\right) x \quad\left(\$ d l_{-} p u s h \_b a s i c\right)
\end{aligned}
$$

Here, the program $d l \_p u s h \_b a s i c_{\text {spec }}$ pushes an element at the end of the list, assuming that there is enough capacity; and the program $d l_{-}$double $e_{\text {spec }}$ doubles the capacity of the dynamic list. The abstract currency $d l_{-}$push_basic represents the costs incurred to push an element and the abstract

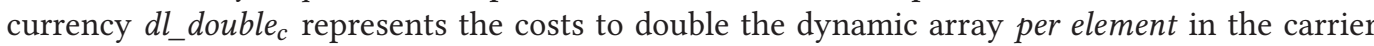
list.

Let us examine the raw, i. e., non-amortized, costs of the operation. If there is capacity left, we have to pay for the if-branch and its guard, as well as the basic push operation. This can be summarized in the constant cost $d l_{\_}$push incurs: $d l_{-}$push_overhead $d_{\text {cost }}=\$_{\text {less }}+\$_{i f}+\$_{d l_{-} p u s h \_b a s i c}$. In the other case, we have to additionally pay for the doubling: push_overhead $d_{c o s t}+\$_{d l_{-} d o u b l e_{c}} c$. Thus, the worst-case cost of the operation is not constant, but rather linear in $c$ because of the double operations.

\footnotetext{
${ }^{21}$ The relation oarr, described in earlier work [24, Section 4.2] by one of the authors, is used to model ownership of parts of a list on an abstract level and is an example for a relation that is not single-valued.
} 
As a next step, we will see how we can formalize the potential method on the NREST level and prove that the abstract push operation has amortized constant time.

\subsection{Amortized Analysis}

The potential method for amortized complexity has the following well-known inequality that relates the raw cost of an operation with its advertised cost and the potential of the data structure before and after an operation.

$$
\text { raw_cost }{ }_{i} \leq\left(\Phi_{i}+\text { advertised_cost } t_{i}\right)-\Phi_{i+1}
$$

Before executing an operation we can get the resource credits from the potential of the data structure and add it to the cost that is advertised to the caller of the operation. Then, we execute the operation incurring the raw costs, and afterwards we need to give back the resource credits for the potential of the resulting data structure. Finally, we can execute several operations on the data structure one after the other and use telescoping to obtain the following inequality

$$
\sum_{0 \leq i<n} \text { raw_cost }_{i} \leq \sum_{0 \leq i<n} \text { advertised_cost } t_{i}
$$

Here, we assume that each raw_cost $t_{i}$ and $\Phi_{i}$ is non-negative and the potential $\Phi_{0}$ is initially zero. The inequality expresses that the real costs are upper bounded by the sum of the advertised costs.

We cannot use elapse to model the subtraction in the amortization inequality, as this would require negative costs. ${ }^{22}$ Instead, we introduce a new combinator reclaim and formulate the amortization inequality in the NREST-monad with an amortization refinement lemma:

$$
\left.m_{\text {raw }} d s \leq \text { reclaim (elapse }\left(m_{a d v} d s\right)(\Phi d s)\right)\left(\lambda d s^{\prime} . \Phi d s^{\prime}\right)
$$

Here, the raw monadic program $m_{\text {raw }}$ executed on some data structure $d s$ has to refine the program that first consumes the potential of the data structure, then executes the monadic program with advertised costs, and in the end reclaims as much costs as the resulting data structure $d s^{\prime}$ needs for its potential.

The combinator reclaim subtracts cost from a monadic program, and fails if it would get negative. Note that this approach only works if the resource type provides a minus operator, as ecost does in our case. Here is the formal definition:

$$
\begin{aligned}
& \text { reclaim }::(\alpha \text {, ecost) NREST } \rightarrow(\alpha \rightarrow \text { ecost }) \rightarrow(\alpha \text {, ecost }) \text { NREST } \\
& \text { reclaim fail } T=\text { fail } \\
& \text { reclaim }(\text { res } M) T=\text { Sup }\left\{\text { if } T x \leq t^{\prime} \text { then res }\left[x \mapsto t^{\prime}-T x\right] \text { else fail } \mid t^{\prime} x . M x=\text { Some } t^{\prime}\right\}
\end{aligned}
$$

For each possible result $x$ of $M$ the combinator checks whether the consumed time $t^{\prime}$ is at least the reclaimed time $T x$ for that result. This ensures not falling into the negative when subtracting. If one of the inequalities does not hold, the whole program reclaim $m t$ fails.

Using reclaim we can state the amortization refinement lemma for $d l \_p u s h$ :

$$
\left.d l \_p u s h d l x \leq \text { reclaim (elapse }\left(d l \_p u s h_{s p e c} d l x\left(p u s h \_a d v_{c o s t}\right)\right)\left(\Phi_{\mathrm{dl}} d l\right)\right)\left(\lambda d l^{\prime} . \Phi_{\mathrm{dl}} d l^{\prime}\right)
$$

Setting $\Phi_{\mathrm{dl}}(c s, l, c)=\$_{d_{1} l_{\text {double }}}(2 * l-c)$ and push_adv $v_{\text {cost }}=$ push_overhead $_{\text {cost }}+\$_{\text {dl_double }_{c}} 2$, our VCG can automatically prove this lemma. ${ }^{23}$

In particular, we have shown that $d l_{-}$push has amortized constant time, as its advertised cost only consumes the push_overhead $d_{\text {cost }}$ and two additional $\$$ dl_double $_{c}$ coins for loading the potential. This argument is independent from how exactly $d l_{-} d o u b l e$ is implemented and how the currency

\footnotetext{
${ }^{22}$ Extending NREST to allow negative costs might streamline the theory. We leave further investigation to future work.

${ }^{23}$ To help us with finding the correct terms for $\Phi_{\mathrm{dl}}$ and $p u s h_{-} a d v_{\text {cost }}$, we can run our VCG with symbolic variables first, and examine the generated proof obligations, which show us the constraints that $\Phi_{\mathrm{dl}}$ and push_adv cost must satisfy.
} 
$\$_{d l_{-} \text {double }_{c}}$ is refined later. That way we achieved to separate the amortization argument from the implementation details.

This already concludes the verification on the NREST-level. We have shown that we can use the potential $\Phi_{\mathrm{dl}}$ to prove $d l_{-}$push having amortized constant time. We can go on proving correct other operations on the data structure with amortization, e. g., lookup, write within bounds, initialization, and destruction. That includes to show that they respect the change of potential. We can also apply telescoping on this level and sequentially compose several reclaim-elapse pairs on the same data structure following the intuition above.

It is left to show that we can actually implement the operation with a concrete program and obtain the desired synthesis rule mentioned in Example 4.1.

\subsection{Moving Potential to Time Credits}

Now we have obtained a refinement in the reclaim-elapse pattern. In order to obtain the desired synthesis rule, we will move the potential from the abstract NREST-program into the pre- and postheap in the synthesis rule. This will only leave the advertised cost in the abstract program.

On the separation logic level we can augment assertions representing raw data structures with time credits representing their potential. The operator [ $\Phi] A r r_{a}=\$ \Phi r_{a} \star A r r_{a}$ adds the potential as time credits depending on the abstract result to an assertion.

Given a synthesis rule that refines a reclaim-elapse pattern we can move the consumed prepotential into the precondition and the reclaimed postpotential into the assertion of the result.

$$
\begin{aligned}
& \left.\left(m_{\dagger}, \lambda(x, r) \text {. reclaim (consume }(m x r)(\Phi x)\right) \Phi\right) \in A^{d} \rightarrow A_{R}{ }^{k} \rightarrow A \\
& \Longrightarrow\left(m_{\dagger}, m\right) \in([\Phi] A)^{d} \rightarrow A_{R}{ }^{k} \rightarrow[\Phi] A
\end{aligned}
$$

Here, the first parameter (called $x$ in the abstract program) is the amortized data structure that is altered and returned as the result. The second parameter (called $r$ in the abstract program) represents the rest of the parameters. They are not modified in this case and do not contribute with amortized potential. We call this rule an amortization synthesis rule. Note that, for simplicity, we have not shown the side conditions that ensure finiteness of the potential and non-failure of the abstract program.

Using that rule the amortization can be moved from the NREST level into the separation logic assertion. The synthesis rule now directly relates the implementation $m_{+}$and the monadic program $m$. In the following, we will explain how this is applied to our example.

\subsection{Obtaining a Synthesis Rule}

In order to obtain a synthesis rule for list_push, we first need to provide an implementation and connect it to the program $d l_{-} p u s h$. Observe that $d l_{-} p u s h$ lives in the currency system of dynamic lists and not of LLVM currencies. We need to refine it to some abstract program $d a \_p u s h$ that fixes the way we implement the carrier list to arrays and refines all operations to operations we have synthesis rules for. This involves exchanging the currencies from dynamic lists to LLVM currencies via some exchange rate $E_{d a}$. In particular, $E_{d a}$ has to specify how the coin $\$_{d_{1} l_{d} \text { ouble }}$ must be exchanged. Those costs will contain the costs for allocating the new carrier list and copying the elements to the new carrier list. Note that those costs need to be specified per element of the original carrier list. For presentation purposes we skip the details of that part and assume we come up with a program $d a \_p u s h$ and a suitable refinement $d a \_p u s h d l x \leq \Downarrow_{C} E_{d a}\left(d l \_p u s h d l x\right)$.

Furthermore, let $d a_{-} r w_{A}$ be the refinement assertion that relates a concrete representation of a dynamic array with a dynamic list holding natural numbers. While the theory is not dependent on the type of the payload, we choose a fixed one here for presentation purposes. We later want to model strings of characters with the dynamic array. So, the concrete part of the assertion $d a \_r a w_{A}$ 
is a triple, consisting of an array of 8 bit integers $\left(\langle 8\rangle\right.$ unat $\left._{A}\right)$ and two 64 bit integers $\left(\langle 64\rangle\right.$ snat $\left._{A}\right)$ for the length and capacity. Further, we assume that we have synthesized an LLVM program $d a_{-} p u s h_{\dagger}$ that refines da_push, with the following synthesis rule:

$$
\left(d a \_p u s h_{\dagger}, d a \_ \text {push }\right) \in\left(\text { da_raw }_{A}\right)^{d} \rightarrow\langle 8\rangle \text { unat }_{A}{ }^{k} \rightarrow \text { da_raw }_{A}
$$

Now we can combine the currency refinement rule for $d a \_p u s h$ and the amortization refinement rule for $d l \_p u s h$ and obtain to the following refinement:

$$
\left.d a \_p u s h d l x \leq \text { reclaim (elapse }\left(d l \_p u s h_{\text {spec }} d l x(\text { push_concrete_adv cost })\right)\left(\Phi_{\mathrm{da}} d l\right)\right) \Phi_{\mathrm{da}}
$$

Here, the currency refinement was already distributed over reclaim and elapse. This yields the following two cost functions: push_ad $v_{\text {cost }}^{\prime}=\downarrow_{C} E_{d a}$ push_advcost and $\Phi_{\mathrm{da}} d l=\downarrow_{C} E_{d a}\left(\Phi_{\mathrm{dl}} d l\right)$. Here, the operation $\downarrow_{C} E t$ applies an exchange rate to a resource function. In particular, as the exchange rate $E_{d a}$ is independent of the dynamic list and push_adv cost is constant, also the advertised cost push_adv $v_{\text {cost }}^{\prime}$ is constant.

We can now combine that refinement rule with the synthesis rule from above. Note that the refinement does not involve data refinement, and thus does not have any attains_sup side conditions (cf. Section 4.5). We obtain the following synthesis rule:

$$
\begin{aligned}
& \left(\lambda\left(d a, x_{\dagger}\right) . d a_{-} p u s h_{\dagger} d a x_{\dagger},\right. \\
& \left.\left.\lambda(d l, x) \text {.reclaim (elapse }\left(d l \_p u s h_{\text {spec }} d l x\left(\text { push_ad } v_{\text {cost }}^{\prime}\right)\right)\left(\Phi_{\mathrm{da}} d l\right)\right) \Phi_{\mathrm{da}}\right) \\
& \in d a \_r a w_{A}{ }^{d} \rightarrow\langle 8\rangle \text { unat }_{A}{ }^{k} \rightarrow d a \_r a w_{A}
\end{aligned}
$$

This form fits the precondition of the amortization synthesis rule, and we can apply it to move the elapsed and reclaimed resources to the pre-heap and the refinement assertion for the result, respectively.

$$
\begin{aligned}
& \left(\lambda\left(d a, x_{\dagger}\right) . d a \_p u s h_{\dagger} d a x_{\dagger}, \lambda(d l, x) d l_{-} p u s h_{s p e c} d l x\left(p u s h \_a d v_{c o s t}^{\prime}\right)\right) \\
& \in\left(\left[\Phi_{\mathrm{da}}\right] d a \_r a w_{A}\right)^{d} \rightarrow\langle 8\rangle \text { unat }_{A}{ }^{k} \rightarrow\left[\Phi_{\mathrm{da}}\right] d a \_r a w_{A}
\end{aligned}
$$

At this point we already have established a refinement between the push operation on dynamic lists $d l_{-} p u s h_{\text {spec }}$ and the implementation on dynamic arrays $d a \_p u s h_{\dagger}$. We could extract a Hoare triple from the synthesis rule that shows the correctness of the implementation and the amortized constant running time.

As a last step, we hide the intermediate concept of dynamic lists and obtain a refinement between the list operation and the implementation on dynamic arrays. First, consider the data refinement between $d l_{\text {_push }}$ and list_push $_{\text {spec }}$. We repeat it here:

$$
\left(d l_{-} \text {push } h_{\text {spec }}(t), \text { list_push }_{\text {spec }}(t)\right) \in R_{\text {dynlist }}^{\text {list }} \rightarrow I d \rightarrow R_{\text {dynlist }}^{\text {list }}
$$

We can apply this data refinement to the synthesis rule above, and use the fact that $R_{d y n l i s t}^{\text {list }}$ is single-valued ${ }^{24}$ to solve the sup-attains side condition. Then, we obtain the final synthesis rule:

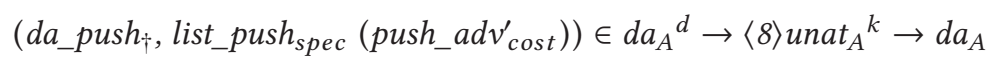

where $d a_{A}$ relates a list with a dynamic array. This refinement assertion combines the refinement relation $R_{d y n l i s t}^{l i s t}$, the raw refinement assertion $d a_{-} r a w_{A}$ and the augmentation with the time credits containing the potential. Formally we define:

$$
d a_{A} \text { as } a l=\exists_{A} d l .\left[\Phi_{\mathrm{da}}\right]\left(d a \_r a w_{A}\right) d l a l \star \uparrow\left((d l, a s) \in R_{\text {dynlist }}^{\text {list }}\right)
$$

\footnotetext{
${ }^{24}$ That is, every dynamic list has at most one corresponding abstract list.
} 
As mentioned at the beginning of this section, for presentation purposes we have left out size constraints that are necessary to avoid overflows in the LLVM implementation. When doubling the list we have to make sure that the multiplication of the capacity with 2 does not lead to an overflow. We can restrict this by adding a size constraint to the synthesis rule demanding the length of the list may at most be half of MAX_INT before pushing an element to it. In a program that uses that operation, one then has to add assertions before those invocations that help the Sepref tool to discharge the respective size constraints. Those size constraints then can be propagated to the precondition of the program. For example, a depth-first search that uses a dynamic array to represent its waiting list might have an additional size constraint restricting the number of edges in the graph to MAX_INT/2.

Once we have the last synthesis rule, we can cut out the whole reasoning with the combinators reclaim and elapse and inspect the rule on its own. The refinement assertion $d a_{A}$ serves as a black box for the user. For a user of the rule, only the constant advertised cost is visible in push_adv $v_{\text {cost }}$ and the whole amortization is hidden and happens under the hood, such that this amortized data structure behaves like any other data structure.

\subsection{Discussion}

Previously, we had to prove amortized data structures on the low-level separation logic (e. g., [14, Section 5.1]), while we can now structure our proofs using the same top-down refinement approach as for non-amortized complexity analysis.

While we have demonstrated our method for the quite simple dynamic array data structure, we believe that more involved amortized analyses can also profit from this technique. A next step would be to modularize the verification of Union-Find [6, 28].

Another advantage of performing the analysis on the abstract NREST-level is the independence from the actual back end. E.g., we could ${ }^{25}$ use the same abstract proof to verify implementations in LLVM and Imperative HOL.

To summarize the refinement process for this case study reconsider Table 1 . We started from a specification of the abstract operation ( list_push $_{\text {spec }}$ ), which can be expressed in the NREST-monad. Then, we data-refined lists to dynamic lists $\left(d l_{-} p u s h_{s p e c}\right)$. We introduced the algorithmic idea as an NREST program $d l_{-}$push using only the specification of abstract operations like $d l_{-} d o u b l e_{\text {spec }}$. Proving the algorithmic idea and amortization argument happens on that level of abstraction. Towards implementing the algorithm, we then refined the abstract operations to basic operations that have available synthesis rules. In that process, we had to use currency refinements to exchange to LLVM currencies in the program $d a \_p u s h$. Finally, we used the Sepref tool to synthesize an LLVM implementation $d a \_p u s h_{\dagger}$, which uses imperative arrays. By transitivity, the refinement chain yields the final synthesis rule relating $l i s t_{-} p u s h_{s p e c}$ and $d a_{-} p u s h_{\dagger}$. The refinement approach allows to separate concerns and address proof obligations on the most abstract and appropriate level.

\section{CASE STUDY: INTROSORT}

In this section, we apply our framework to the introsort algorithm [30]. We build upon the verification of its functional correctness [24] to verify its running time analysis and synthesize competitive efficient LLVM code for it. Following the "top-down" mantra, we use several intermediate steps to refine a specification down to an implementation.

\footnotetext{
${ }^{25}$ In practice, we have to copy and slightly adjust the proof, as the front-ends for LLVM and Imperative HOL are not yet unified.
} 


\subsection{Specification of Sorting}

We start with the specification of sorting a slice of a list:

$$
\begin{aligned}
& \text { slice_sort }_{\text {spec }} x s_{0} l h(t)= \\
& \text { assert }\left(l \leq h \wedge h \leq\left|x s_{0}\right|\right) ; \\
& \text { spec }\left(\lambda x s \text {. slice_sort_aux } x s_{0} l h x s\right)\left(\lambda \_t\right)
\end{aligned}
$$

where slice_sort_aux $x s_{0} l h x s$ states that $x s$ is a permutation of $x s_{0}, x s$ is sorted between $l$ and $h$ and equal to $x s_{0}$ anywhere else.

\subsection{Introsort's Idea}

The introsort algorithm is based on quicksort. Like quicksort, it finds a pivot element, partitions the list around the pivot, and recursively sorts the two partitions. Unlike quicksort, however, it keeps track of the recursion depth, and if it exceeds a certain value (typically $\lfloor 2 \log n\rfloor$ ), it falls back to heapsort to sort the current partition. Intuitively, quicksort's worst-case behavior can only occur when unbalanced partitioning causes a high recursion depth, and the introsort algorithm limits the recursion depth, falling back to the $O(n \log n)$ heapsort algorithm. This combines the good practical performance of quicksort with the good worst-case complexity of heapsort.

Our implementation of introsort follows the implementation of libstdc++, which includes a second optimization: a first phase executes quicksort (with fallback to heapsort), but stops the recursion when the partition size falls below a certain threshold $\tau$. Then, a second phase sorts the whole list with one final pass of insertion sort. This exploits the fact that insertion sort is actually faster than quicksort for almost-sorted lists, i.e., lists where any element is less than $\tau$ positions away from its final position in the sorted list. While the optimal threshold $\tau$ needs to be determined empirically, it does not influence the worst-case complexity of the final insertion sort, which is $O(\tau n)=O(n)$ for constant $\tau$. The threshold $\tau$ will be an implicit parameter from now on.

While this seems like a quite concrete optimization, the two phases are already visible in the abstract algorithm, which is defined as follows in NREST:

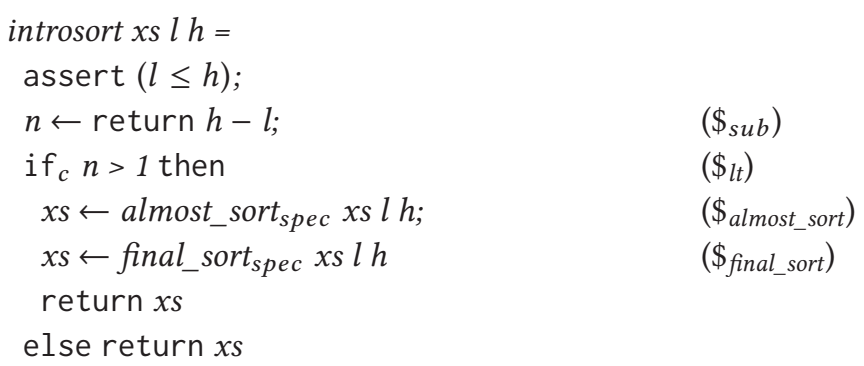

Here, almost_sort $t_{\text {spec }}(t)$ specifies an algorithm that almost-sorts a list, consuming at most $t$ resources and final_sort spec $(t)$ specifies an algorithm that sorts an almost-sorted list, consuming at most $t$ resources.

The program introsort leaves trivial lists unchanged and otherwise executes the first and second phase. Its resource usage is bounded by the sum of the first and second phase and some overhead for the subtraction, comparison, and if-then-else. Using the verification condition generator we prove that introsort is correct, i.e., refines the specification of sorting a slice:

introsort xs $l h \leq \Downarrow_{C} E_{i s}\left(\right.$ slice_sort $_{\text {spec }}$ xs $\left.l h\left(\$_{\text {sort }}\right)\right)$

where $E_{i s}=\uparrow \downarrow\left[\right.$ sort $:=$ introsort $\left._{\text {cost }}\right]$ is the exchange rate used at this step and the total allotted cost for introsort is introsort $t_{\text {cost }}=\$_{\text {sub }}+\$_{\text {if }}+\$_{\text {lt }}+\$_{\text {almost_sort }}+\$_{\text {final_sort }}$. 


\subsection{Introsort Scheme}

The first phase can be implemented in the following way:

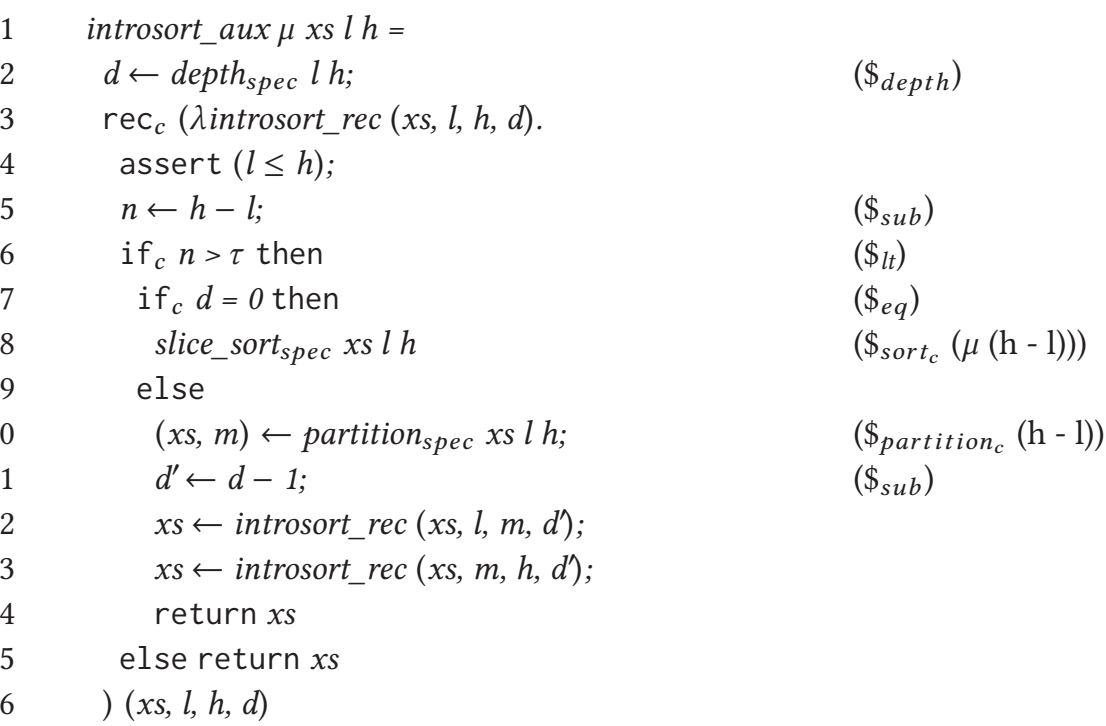

where partition $_{\text {spec }}$ partitions a slice into two non-empty partitions, returning the start index $m$ of the second partition, and depth $h_{\text {spec }}$ specifies $\lfloor 2 \log (h-l)\rfloor$.

Let us first analyze the recursive part: if the slice is shorter than the threshold $\tau$, it is simply returned (line 15). Unless the recursion depth limit is reached, the slice is partitioned using $h-l$ partition $_{c}$ coins, and the procedure is called recursively for both partitions (lines 10-14). Otherwise, the slice is sorted at a price of $\mu(h-l)$ sort $_{c}$ coins (line 8). The function $\mu$ here represents the leading term in the asymptotic costs of the used sorting algorithm, and the sort $t_{c}$ coin can be seen as the constant factor. This currency will later be exchanged into the respective currencies that are used by the sorting algorithm. Note that we use currency sort $t_{c}$ to describe costs per comparison of a sorting algorithm, while currency sort describes the cost for a whole sorting algorithm.

Showing that the procedure results in an almost-sorted list is straightforward. The running time analysis, however, is a bit more involved. We presume a function $\mu$ that maps the length of a slice to an upper bound on the abstract steps required for sorting the slice. We will later use heapsort with $\mu_{n \log n} n=n \log n$.

Consider the recursion tree of a call in introsort_rec: We pessimistically assume that for every leaf in the recursion tree we need to call the fallback sorting algorithm. Furthermore, we have to partition at every inner node. This has cost linear in the length of the current slice. For each following inner level the lengths of the slices add up to the current one's, and so do the incurred costs. Finally, we have some overhead at every level including the final one. The cost of the recursive part of introsort_aux is:

$$
\begin{aligned}
& \text { introsort_rec }_{\text {cost }} \mu(n, d)=\$_{\text {sort }_{c}}(\mu n)+\$_{\text {partition }_{c}} d * n \\
& +((d+1) * n) *\left(\$_{i f} 2+\$_{\text {call }} 2+\$_{\text {eq }}+\$_{\text {lt }}+\$_{\text {sub }} 2\right)
\end{aligned}
$$

The correctness of the running time bound is proved by induction over the recursion of introsort_rec. If the recursion limit is reached $(d=0)$, the first summand pays for the fallback sorting algorithm. If $d>0$, part of the second summand pays for the partitioning of the current slice, then the list is split into two and the recursive costs are payed for by parts of all three summands. To 
bound the costs for the fallback sorting algorithm, $\mu$ needs to be superadditive: $\mu a+\mu b \leq \mu(a+b)$. In both cases, the third summand pays for the overhead in the current call.

For $d=\lfloor 2 \log n\rfloor$ and an $O(n \log n)$ fallback sorting algorithm $\left(\mu=\mu_{n \log n}\right)$, introsort_rec cost $_{\text {in }} \mu_{n \log n}$ is in $O(n \log n) .{ }^{26}$ In fact, any $d \in O(\log n)$ would do.

Before executing the recursive method, introsort_aux calculates the depth limit $d$. The correctness theorem then reads:

introsort_aux $\mu_{n l o g n} x s l h \leq \Downarrow_{C}\left(E_{i s a}(h-l)\right)\left(\right.$ almost_sort $\left._{\text {spec }} x s l h\left(\$_{\text {almost_sort }}\right)\right)$

Where $E_{\text {isa }} n=\uparrow \downarrow\left[\right.$ almost_sort $:=\$_{\text {depth }}+$ introsort_rec cost $\left.\mu_{n \log n}(n,\lfloor 2 \log n\rfloor)\right]$.

Note that specifications typically use a single coin of a specific currency for their abstract operation, which is then exchanged for the actual costs, usually depending on the parameters.

This concludes the interesting part of the running time analysis of the first phase. It is now left to plug in an $O(n \log n)$ fallback sorting algorithm, and a linear partitioning algorithm.

Heapsort. Independently of introsort, we have proved correctness and worst-case complexity of heapsort, yielding the following refinement lemma:

heapsort xs $l h \leq \Downarrow_{C}\left(E_{h s}(h-l)\right)\left(\right.$ slice_sort $\left._{\text {spec }} x s l h\left(\$_{\text {sort }}\right)\right)$

Where $E_{h s} n=\uparrow \downarrow\left[\right.$ sort $\left.:=c_{1}+\log n * c_{2}+n * c_{3}+(n * \log n) * c_{4}\right]$ for some constants $c_{i}::$ ecost.

Assuming that $n \geq 2,{ }^{27}$ we can estimate $E_{h s} n$ sort $\leq \mu_{n l o g n} n * c$, for $c=c_{1}+c_{2}+c_{3}+c_{4}$, and thus get, for $E_{h s^{\prime}}=\uparrow \downarrow\left[\operatorname{sort}_{c}:=c\right]$ :

$$
\begin{aligned}
& \Downarrow_{C}\left(E_{h s}(h-l)\right)\left(\text { slice_sort }_{\text {spec }} x s l h\left(\$_{\text {sort }}\right)\right) \\
& \leq \Downarrow_{C} E_{h s^{\prime}}\left(\text { slice_sort }_{\text {spec }} x s l h\left(\$_{\text {sort }}\left(\mu_{\text {nlogn }}(h-l)\right)\right)\right)
\end{aligned}
$$

and, by, transitivity

heapsort xs $l h \leq \Downarrow_{C} E_{h s^{\prime}}\left(\right.$ slice_sort $\left._{\text {spec }} x s l h\left(\$_{\text {sort }}\left(\mu_{n l o g n}(h-l)\right)\right)\right)$

Note that our framework allowed us to easily convert the abstract currency from a single operationspecific sort coin to a sort $c$ coin for each comparison operation.

Partition and Depth Computation. We implement partitioning with the Hoare partitioning scheme using the median-of- 3 as the pivot element. Moreover, we implement the computation of the depth limit $(2\lfloor\log (h-l)\rfloor)$ by a loop that counts how often we can divide by two until zero is reached. This yields the following refinement lemmas:

pivot_partition xs $l h \leq \Downarrow_{C} E_{p p}\left(\right.$ partition $\left._{\text {spec }} x s l h\left(\$_{\text {partition }_{c}}(h-l)\right)\right)$

calc_depth $l h \leq \Downarrow_{C}\left(E_{c d}(h-l)\right)\left(\right.$ depth $\left._{\text {spec }} l h\left(\$_{\text {depth }}\right)\right)$

Combining the Refinements. We replace slice_sort $_{\text {spec }}$, partition spec $_{\text {and }}$ aepth $h_{\text {spec }}$ by their implementations heapsort, pivot_partition and calc_depth. Finally, we call the resulting implementation introsort_aux 2 , and prove

introsort_aux $2 x s l h \leq \Downarrow_{C}\left(E_{\text {aux }}(h-l)\right)\left(\right.$ introsort_aux $\left.\mu_{n l o g n} x s l h\right)$

Where the exchange rate $E_{a u x}$ combines the exchange rates $E_{h s^{\prime}}, E_{p p}$ and $E_{c d}$ for the component refinements.

Transitive combination with the correctness lemma for introsort_aux then yields the correctness lemma for introsort_aux $x_{2}$ :

\footnotetext{
${ }^{26}$ More precisely, the sum over all (finitely many) currencies is in $O(n \log n)$.

${ }^{27}$ Note that this is a valid assumption, as heapsort will never be called for trivial slices.
} 
introsort_aux $_{2} x s l h \leq \Downarrow_{C}\left(E_{\text {isa2 }}(h-l)\right)\left(\right.$ almost_sort $\left._{\text {spec }} x s l h\left(\$_{\text {almost_sort }}\right)\right)$

Where $E_{\text {isa2 }} n=\uparrow \downarrow\left[\right.$ almost_sort $:=\downarrow_{C}\left(E_{\text {aux }} n\right)$ (introsort_aux cost $\left.\left.n\right)\right]$ and the operation $\downarrow_{C} E t$ applies an exchange rate to a resource function.

Refining Resources. The stepwise refinement approach allows to structure an algorithm verification in a way that correctness arguments can be conducted on a high level and implementation details can be added later. Resource currencies permit the same for the resource analysis of algorithms: they summarize compound costs, allow reasoning on a higher level of abstraction and can later be refined into fine-grained costs. For example, in the resource analysis of introsort_aux the currencies sort $_{c}$ and partition ${ }_{c}$ abstract the cost of the respective subroutines. The abstract resource argument is independent from their implementation details, which are only added in a subsequent refinement step, via the exchange rate $E_{\text {aux }}$.

\subsection{Final Insertion Sort}

The second phase is implemented by insertion sort, repeatedly calling the subroutine insert. The specification of insert for an index $i$ captures the intuition that it goes from a slice that is sorted up to index $i-1$ to one that is sorted up to index $i$. Insertion is implemented by moving the last element to the left, as long as the element left of it is greater (or the start of the list has been reached). Moving an element to its correct position takes at most $\tau$ steps, as after the first phase the list is almost-sorted, i.e., any element is less than $\tau$ positions away from its final position in the sorted list. Moreover, elements originally at positions greater $\tau$ will never reach the beginning of the list, which allows for the unguarded optimization. It omits the bounds check for those elements, saving one index comparison in the innermost loop. Formalizing these arguments yields the implementation final_insertion_sort that satisfies

$$
\text { final_insertion_sort xs } l h \leq \Downarrow_{C}\left(E_{f i s}(h-l)\right)\left(\text { final_sort }_{\text {spec }} x s l h\left(\$_{\text {final_sort }}\right)\right)
$$

Where $E_{f i s} n=\uparrow \downarrow\left[\right.$ final_sort :=final_insertion cost $\left._{\text {c }} n\right]$, and final_insertion cost $_{n} n$ is linear in $n$.

Note that final_insertion_sort and introsort_aux 2 use the same currency system. Plugging both refinements into introsort yields introsort 2 and the lemma

introsort $_{2}$ xs $l h \leq \Downarrow_{C}\left(E_{i s 2}(h-l)\right)($ introsort xs $l h)$

Where the exchange rate $E_{i s 2}$ combines the rates $E_{i s a 2}$ and $E_{f i s}$.

\subsection{Separating Correctness and Complexity Proofs}

A crucial function in heapsort is sift_down, which restores the heap property by moving the top element down in the heap. To implement this function, we first prove correct a version sift_down $n_{1}$, which uses swap operations to move the element. In a next step, we refine this to sift_down $n_{2}$, which saves the top element, then executes upward moves instead of swaps, and, after the last step, moves the saved top element to its final position. This optimization spares half of the memory accesses, exploiting the fact that the next swap operation will overwrite an element just written by the previous swap operation.

However, this refinement is not structural: it replaces swap operations by move operations, and adds an additional move operation at the end. At this point, we chose to separate the functional correctness and resource aspect, to avoid the complexity of a combined non-structural functional and currency refinement. It turns out that proving the complexity of the optimized version sift_down $n_{2}$ directly is straightforward. Thus, as sketched in Section 2.6, we first prove ${ }^{28}$

\footnotetext{
${ }^{28}$ Note that we have omitted the function parameters for better readability.
} 
sift_down $n_{2} \leq$ sift_down $n_{1} \leq$ sift_down $n_{\text {spec }}(\infty)$, ignoring the resource aspect. Separately, we prove sift_down ${ }_{2} \leq_{n} \operatorname{spec}\left(\lambda_{\text {. }}\right.$ True $)$ sift_down $n_{\text {cost }}$, and combine the two statements to get the final refinement lemma:

$$
\text { sift_down } 2 \leq \text { sift_down }
$$

\subsection{Refining to LLVM}

To obtain an LLVM implementation of our sorting algorithm, we have to specify an implementation for the data structure that holds the elements, and for the comparison operator on elements. We use arrays for the data structure, and parameterize over the comparison function (see Section 6.7). Let $E_{3}$ be the corresponding exchange rate from abstract data structure access and comparison to actual LLVM operations. We obtain introsort $_{3} x s l h \leq \Downarrow_{C} E_{3}$ (introsort ${ }_{2} x s l h$ ), and can automatically synthesize an LLVM program introsort ${ }_{\uparrow}$ that refines introsort in $_{3}$, i.e., satisfies the theorem:

$$
\left(\text { introsort }_{\uparrow}, \text { introsort }_{3}\right) \in \text { array }_{A}{ }^{d} \rightarrow \text { snat }_{A}{ }^{k} \rightarrow \text { snat }_{A}{ }^{k} \rightarrow \text { array }_{A}
$$

Combination with the refinement lemmas for introsort introsort $_{2}$, and introsort, followed by conversion to a Hoare triple, yields our final correctness statement:

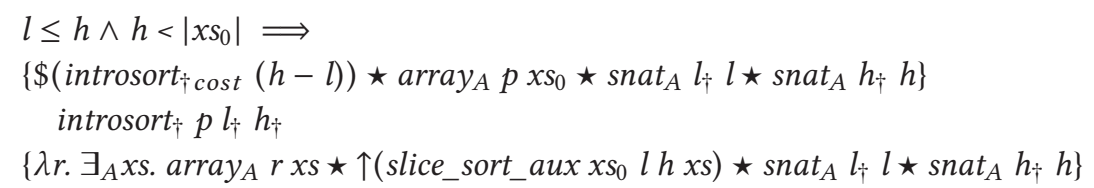

Where introsort $_{\dagger \text { cost }}::$ nat $\rightarrow$ ecost is the cost bound obtained from applying the exchange rates $E_{i s}$, then $E_{i s 2}$, and finally $E_{3}$ to $\$$ sort .

Note that this statement is independent of the Refinement Framework. Thus, to believe in its meaningfulness, one has to only check the formalization of Hoare triples, separation logic, and the LLVM semantics.

To formally prove the statement "introsort ${ }_{\dagger}$ has complexity $O(n \log n)$ ", we first observe that introsort $_{\uparrow \text { cost }}$ uses only finitely many currencies, and only finitely many coins of each currency. Then, we define the overall number of coins as

$$
\text { introsort }_{\dagger \text { allcost }} n=\sum \text { c. } \text { introsort }_{\dagger \cos t} n c
$$

which expands to

$$
\text { introsort }_{\dagger \text { allcost }} n=4693+5 * \log n+231 * n+455 *(n * \log n)
$$

which, in turn, is routinely proved to be in $O(n \log n)$.

Finally, instantiating the element type and comparison operation yields a complete LLVM program, that our code generator can translate to actual LLVM text and a corresponding header file for interfacing our sorting algorithm from $\mathrm{C}$ or C++. For example, with LLVM's $i 64$ type and the unsigned compare operation $l l_{-} i c m p \_u l t$, we get a program that sorts unsigned 64 bit integers in ascending order.

As LLVM does not support generics, we cannot implement a replacement for C++'s generic $s t d:$ sort. However, by repeating the instantiation for different types and compare operators, we can implement a replacement for any fixed element type.

\subsection{Sorting Strings}

We now elaborate on the parameterization over element types that we described in the last section, and also show how to sort elements with non-constant-time compare operations, such as strings. 


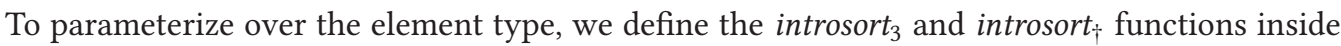
a locale. Locales in Isabelle fix parameters with assumptions that can be instantiated later.

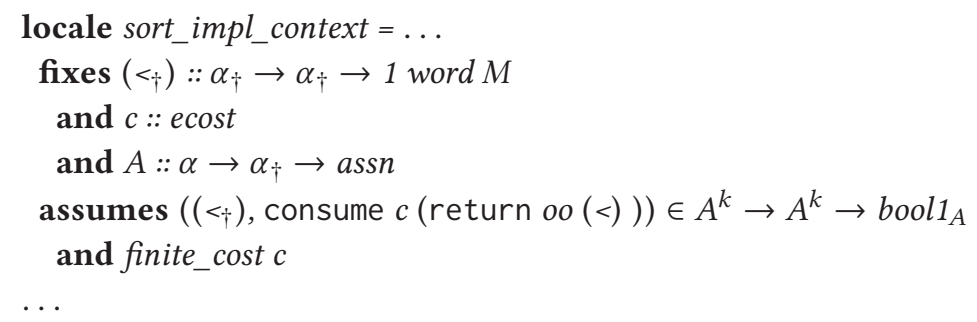

Here, $\alpha$ is the abstract element type, $\alpha_{\dagger}$ is the concrete element type, $<_{\dagger}$ is the implementation of the compare function that requires cost $c$, and $A$ is the refinement relation for elements. The assumptions state that $<$ actually implements the comparison, and that the required costs are finite.

This locale can now be instantiated for different element types. For example, the instantiation to uint64-as described in the previous section-is done as follows:

$$
\text { global_interpretation sort_impl_context... ll_icmp_ult } \$_{i c m p \_u l t}\langle 64\rangle u n a t_{A}
$$

A more complex element datatype is string. It can be implemented by dynamic arrays ${ }^{29}$ (cf. Section 5). In the original formalization without costs, it is straightforward to implement a lexicographic compare operator on dynamic arrays $\left(\operatorname{strcmp} p_{\dagger}\right)$, to show that it refines the lexicographic ordering on lists, and to instantiate the parameterized sorting algorithm.

However, when adding costs, the costs of comparing two strings depend on the lengths of the strings. In our implementation, comparison is linear in the length of the shorter string. This dependency on the input parameters poses a challenge to the analysis of the algorithm. In our formalization, we simply over-estimate the cost for a comparison by the longest string in the array to be sorted. While more precise analyses might be possible, this approach integrates nicely into our existing formalization infrastructure, and still yields usable upper bounds for not too extreme length distributions.

To integrate our over-estimation into the existing formalization, we define an element assertion that contains a maximum length parameter $N$, constraining the length of the strings in the array to at most $N$ :

$$
\operatorname{bstring}_{A} N=\text { bound }_{A}\left(d a_{A}\right)(\lambda x s .|x s|<N)
$$

Here, the assertion bound $A P$ c $a=A c a \star \uparrow(P a)$ restricts an assertion $A$ by a predicate $P$ on the abstract values.

Using this assertion, we can estimate the cost of a string comparison $\left(\operatorname{strcmp} p_{c} N\right)$ to only depend on $N$, and instantiate the algorithm as follows:

$$
\text { global_interpretation sort_impl_context ...strcmp } p_{\dagger}\left(\operatorname{strcmp} p_{c} N\right)\left(b \operatorname{string}{ }_{A} N\right) \text { for } N
$$

While this instantiation is still parametric in $N$, the parameter $N$ does not occur in the implementation, such that we get a fully instantiated implementation which we can export to actual LLVM text. In the final correctness statement, the costs are parameterized over $N$, and we get the estimation:

$$
\text { introsort }_{\dagger \text { allcost }} N n \in O(N * n * \log n)
$$

\footnotetext{
${ }^{29} \mathrm{In} \mathrm{C}++$, the string datatype is typically implemented by a dynamic array, too, however, with some optimizations for short strings, which we omit here.
} 
Discussion. Thanks to Isabelle's locale mechanism, instantiation of our algorithm to an element relation that depends on an extra parameter is pretty straightforward, thus allowing us to also estimate running times for element types with more complex comparison functions, like strings.

Instead of refining the abstract currency for comparing elements to a parametric currency, and then further instantiating the parameters with a concrete implementation, we could also have done the instantiation to element types on the abstract level, and then refined the algorithm to LLVM for each element type. However, our parametric approach saves the overhead of duplicating these refinement steps for each element type.

\subsection{Benchmarks}

In this section, we present benchmarks comparing the code extracted from our formalization with the real world implementation of introsort from the GNU C++ Library (libstdc++). Also, as a regression test, we compare with the code extracted from an earlier formalization of introsort [24] that did not verify the running time complexity and used an earlier iteration of the Sepref framework and LLVM semantics without time.

Ideally, the same algorithm should take exactly the same time when repeatedly run on the same data and machine. However, in practice, we encountered some noise up to $17 \%$. Thus, we have repeated each experiment at least ten times, and more often to confirm outliers where the verified and unverified algorithms' run times differ significantly. Assuming that the noise only slows down an algorithm, we take the fastest time measured over all repetitions. The results are shown in Figure 1. As expected, all three implementations have similar running times. We conclude that adding the complexity proof to our introsort formalization, and the time aspect to our refinement process has not introduced any timing regressions in the generated code. Note, however, that the code generated by our current formalization is not identical to what the original formalization generated. This is mainly due to small changes in the formalization introduced when adding the timing aspect.

\section{CONCLUSIONS}

We have presented a refinement framework for the simultaneous verification of functional correctness and complexity of algorithm implementations with competitive practical performance.

We use stepwise refinement to separate high-level algorithmic ideas from low-level optimizations, enabling convenient verification of highly optimized algorithms. The novel concept of resource currencies allows structuring of the complexity proofs along the refinement chain. Refinement also works seamlessly for amortized data structures. Our framework refines down to the LLVM intermediate representation, such that we can use a state-of-the-art compiler to generate performant programs.

As a case study, we have proved the functional correctness and complexity of the introsort sorting algorithm. Our design supports arbitrary element types, even those with non-constant-time compare operations, like strings. Our verified implementation performs on par with the (unverified) state-of-the-art implementation from the GNU C++ Library. It also provably meets the $\mathrm{C}++11$ standard library [8] specification for std::sort, which in particular requires a worst-case time complexity of $O(n \log n)$. We are not aware of any other verified implementations of real-world sorting algorithms that come with a complexity analysis.

Our work is a combination and substantial extension of an earlier refinement framework for functional correctness [22] which also comes with a verification of introsort [24], and a refinement framework for a single enat-valued currency [14]. In particular, we have generalized the refinement framework to arbitrary resources, applied it to amortized analysis, introduced currencies that help organizing refinement proofs, extended the LLVM semantics and reasoning infrastructure with a 

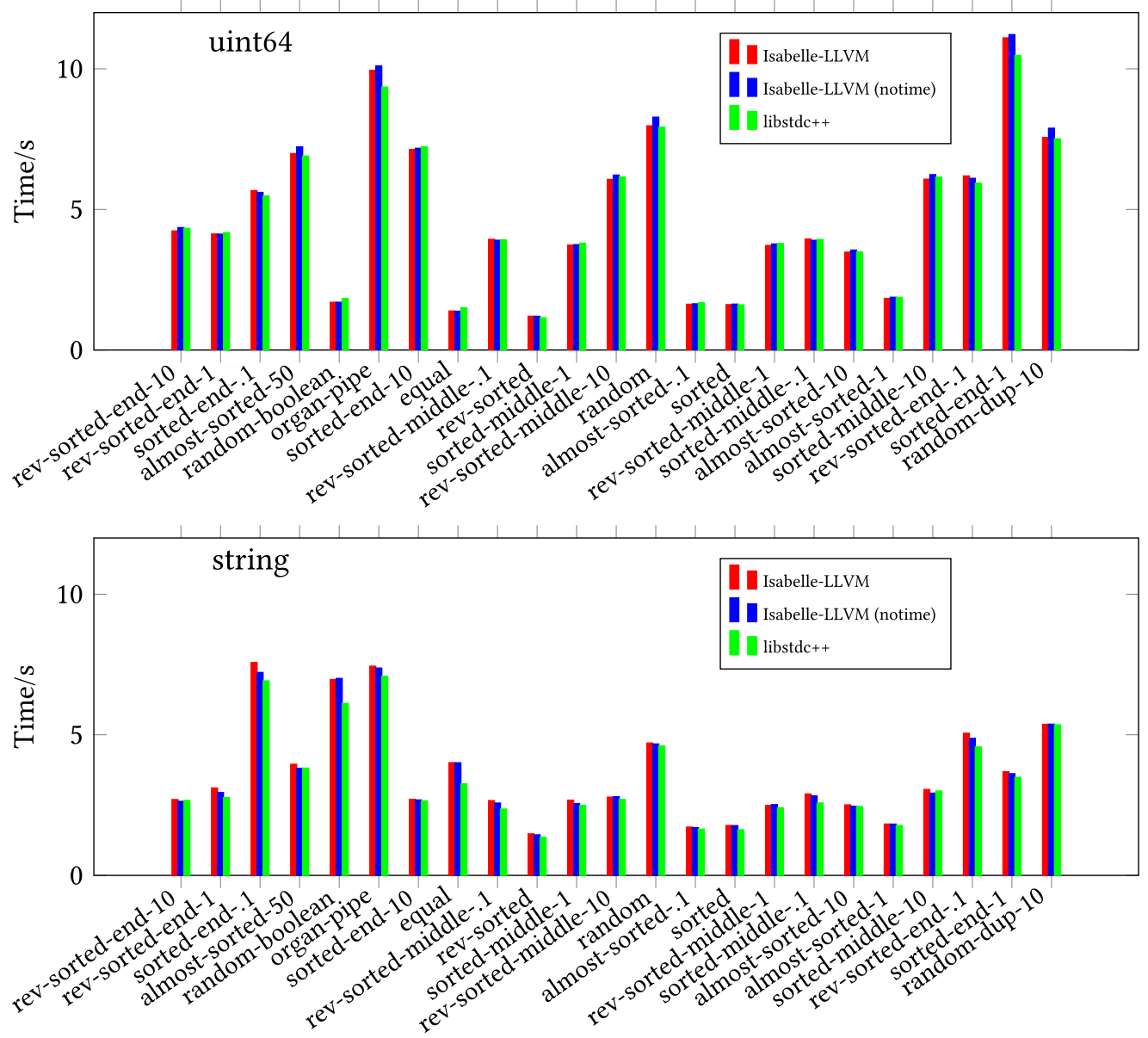

Fig. 1. Comparison of the running time measured for the code generated by the formalization described in this article (Isabelle-LLVM), the original formalization from [24] (notime), and the libstdc++ implementation. Arrays with $10^{8}$ uint64s and $10^{7}$ strings with various distributions were sorted, and we display the smallest time of 10 runs. The programs were compiled with clang-10 -O3, and run on an Intel XEON E5-2699 with 128GiB RAM and 256K/55M L2/L3 cache.

cost model, connected it to the refinement framework via a new version of the Sepref tool, and, finally, added the complexity analysis for introsort.

\subsection{Related Work}

Nipkow et al. [31, Section 4.1] collect verification efforts concerning sorting algorithms. We add a few instances verifying running time: Wang et al. use TiML [36] to verify correctness and asymptotic time complexity of mergesort automatically. Zhan and Haslbeck [37] verify functional correctness and asymptotic running time analysis of imperative versions of insertion sort and mergesort. We build on earlier work by Lammich [24] and provide the first verification of functional correctness and asymptotic running time analysis of heapsort and introsort.

The following are the most complex algorithms and data structures with verified running time analysis using time credits and separation logic we are aware of: a linear time selection algorithm 
[37], an incremental cycle detection algorithm [13], Union-Find [7], Edmonds-Karp and Kruskal's algorithm [14].

The idea to generalize the nres monad [26] to resource types originates from Carbonneaux et al. [4]. They use potential functions (state $\rightarrow$ enat) instead of predicates (state $\rightarrow$ bool), present a quantitative Hoare logic, and extend the CompCert compiler to preserve properties of stackusage from programs in Clight to compiled programs. Observe, that the step from qualitative [9] to quantitative weakest preconditions (cf. Section 2.6) is similar to the weakest preexpectation transformer by Kozen [18], and the expected running time transformer ert by Kaminski et al. [17].

Rajani et al. [33] present a unifying type-theory $\lambda^{\text {amor }}$ for higher-order amortized cost analysis, which involves a cost monad similar to NREST without nondeterminism. The introduction of the elapse combinator is straightforward, but the reclaim operator in NREST seems to be related to their type constructor $[p] \tau$. That constructor is central to their paper. Rajani [32] applies type-theoretic approach to Information Flow Control and generalizes the theory to allow any commutative monoid in the cost monad. It would be interesting to see whether their cost monad can be extended to nondeterminism.

We see our article in the line of research concerning simultaneously verifying functional correctness and worst-case time complexity of algorithms. Atkey [1] pioneered resource analysis with separation logic. Charguéraud and Pottier [6,7] present a framework that uses time credits in Coq and apply it to the Union-Find data structure. Guéneau et al. extend that framework with big-O style specifications [12] and possibly negative time credits, and apply it to involved algorithms and data structures [13]. We further develop their work in three ways: First, while time credits usually are natural numbers $[1,7,12,29,37]$ or integers [13], we generalize to an abstract resource type and specifically use resource currencies for a fine-grained analysis. Second, we use stepwise refinement to structure the verification and make the resource analysis of larger use-cases manageable. Third, we provide facilities to automatically extract efficient competitive code from the verification.

\subsection{Future Work}

A verified compiler down to machine code would further reduce the trusted code base of our approach. While that is not expected to be available soon for LLVM in Isabelle, the NREST-monad and the Sepref tool are general enough to connect to a different back end. Formalizing one of the CompCert $\mathrm{C}$ semantics [2] in Isabelle, connecting it to the NREST-monad and then processing synthesized C code with CompCert's verified compiler would be a way to go.

In this article, we apply our framework to verify an involved algorithm that only uses basic data structures, i.e., arrays. A next step is to verify more involved data structures, e.g., by porting existing verifications of the Imperative Collections Framework [23] to LLVM. We do not yet see how to reason about the running time of data structures like hash maps, where worst-case analysis would be possible but not useful. In general, extending the framework to average-case analysis and probabilistic programs are exciting roads to take.

We plan to implement more automation, saving the user from writing boilerplate code when handling resource currencies and exchange rates.

Neither the LLVM nor the NREST level of our framework is tied to running time. Applying it to other resources like maximum heap space consumption might be a next step.

\section{ACKNOWLEDGMENTS}

We thank Armaël Guéneau, Arthur Charguéraud, François Pottier, and the anonymous referees of ESOP2021 and TOPLAS who provided valuable feedback on the earlier versions of this article. 


\section{REFERENCES}

[1] Robert Atkey. 2010. Amortised resource analysis with separation logic. In Proceedings of the European Symposium on Programming, Andrew D. Gordon (Ed.), Lecture Notes in Computer Science, Vol. 6012, Springer, 85-103. DOI:https: //doi.org/10.1007/978-3-642-11957-6_6

[2] Sandrine Blazy and Xavier Leroy. 2009. Mechanized semantics for the clight subset of the C language. fournal of Automated Reasoning 43, 3 (2009), 263-288. DOI:https://doi.org/10.1007/s10817-009-9148-3

[3] Cristiano Calcagno, Peter W. O’Hearn, and Hongseok Yang. 2007. Local action and abstract separation logic. In Proceedings of the Symposium on Logic in Computer Science. IEEE Computer Society, 366-378. DOI:https://doi.org/10.1109/ LICS.2007.30

[4] Quentin Carbonneaux, Jan Hoffmann, Tahina Ramananandro, and Zhong Shao. 2014. End-to-end verification of stackspace bounds for C programs. In Proceedings of the ACM SIGPLAN Conference on Programming Language Design and Implementation, Michael F. P. O’Boyle and Keshav Pingali (Eds.). ACM, 270-281. DOI:https://doi.org/10.1145/2594291. 2594301

[5] Arthur Charguéraud. 2020. Separation logic for sequential programs (functional pearl). Proceedings of the ACM on Programming Languages 4, ICFP (2020), 116:1-116:34. DOI:https://doi.org/10.1145/3408998

[6] Arthur Charguéraud and François Pottier. 2015. Machine-checked verification of the correctness and amortized complexity of an efficient union-find implementation. In Proceedings of the 6th International Conference on Interactive Theorem Proving Christian Urban and Xingyuan Zhang (Eds.), Lecture Notes in Computer Science, Vol. 9236, Springer, 137-153. DOI:https://doi.org/10.1007/978-3-319-22102-1_9

[7] Arthur Charguéraud and François Pottier. 2019. Verifying the correctness and amortized complexity of a Union-Find implementation in separation logic with time credits. fournal of Automated Reasoning 62, 3 (2019), 331-365. DOI:https //doi.org/10.1007/s10817-017-9431-7

[8] cppreference. [n.d.]. C++ Standard Library Specification of Sort. Retrieved 12 October, 2020 from https://en. cppreference.com/w/cpp/algorithm/sort.

[9] Edsger W. Dijkstra. 1976. A Discipline of Programming. Prentice-Hall. Retrieved from https://www.worldcat.org/oclc/ 01958445.

[10] GNU C++ Library [n.d.]. The GNU C++ Library. Retrieved May 18th, 2022 from https://gcc.gnu.org/onlinedocs/ libstdc++/. Version 7.4.0.

[11] Armaël Guéneau. 2019. Mechanized Verification of the Correctness and Asymptotic Complexity of Programs. (Vérification mécanisée de la correction et complexité asymptotique de programmes). Ph.D. Dissertation. Inria, Paris, France. Retrieved from https://tel.archives-ouvertes.fr/tel-02437532.

[12] Armaël Guéneau, Arthur Charguéraud, and François Pottier. 2018. A fistful of dollars: Formalizing asymptotic complexity claims via deductive program verification. In Proceedings of the 27th European Symposium on Programming, Programming Languages and Systems. Amal Ahmed (Ed.), Lecture Notes in Computer Science, Vol. 10801, Springer, 533-560. DOI:https://doi.org/10.1007/978-3-319-89884-1_19

[13] Armaël Guéneau, Jacques-Henri Jourdan, Arthur Charguéraud, and François Pottier. 2019. Formal proof and analysis of an incremental cycle detection algorithm. In Proceedings of the 10th International Conference on Interactive Theorem Proving. John Harrison, John O’Leary, and Andrew Tolmach (Eds.). Schloss Dagstuhl - Leibniz-Zentrum für Informatik, 18:1-18:20. DOI:https://doi.org/10.4230/LIPIcs.ITP.2019.18

[14] Maximilian P. L. Haslbeck and Peter Lammich. 2019. Refinement with time - refining the run-time of algorithms in Isabelle/HOL. In Proceedings of the 10th International Conference on Interactive Theorem Proving. John Harrison, John O'Leary, and Andrew Tolmach (Eds.). Schloss Dagstuhl - Leibniz-Zentrum für Informatik, 20:1-20:18. DOI:https: //doi.org/10.4230/LIPIcs.ITP.2019.20

[15] Maximilian P. L. Haslbeck and Peter Lammich. 2021. For a few dollars more - verified fine-grained algorithm analysis down to LLVM. In Proceedings of the 30th European Symposium on Programming Languages and Systems. Nobuko Yoshida (Ed.), Lecture Notes in Computer Science, Vol. 12648, Springer, 292-319. DOI:https://doi.org/10.1007/978-3 030-72019-3_11

[16] C. A. R. Hoare. 1961. Algorithm 64: Quicksort. Communication of the ACM 4, 7 (July 1961), 321-. DOI:https://doi.org/ $10.1145 / 366622.366644$

[17] Benjamin Lucien Kaminski, Joost-Pieter Katoen, Christoph Matheja, and Federico Olmedo. 2016. Weakest precondition reasoning for expected run-times of probabilistic programs. In Proceedings of the European Symposium on Programming Languages and Systems. Springer, 364-389.

[18] Dexter Kozen. 1985. A probabilistic PDL. Journal of Computer and System Sciences 30, 2 (1985), 162-178. DOI:https: //doi.org/10.1016/0022-0000(85)90012-1

[19] Alexander Krauss. 2010. Recursive definitions of monadic functions. Electronic Proceedings in Theoretical Computer Science 43 (Dec. 2010), 1-13. DOI: https://doi.org/10.4204/eptcs.43.1 
[20] Peter Lammich. 2015. Refinement to Imperative/HOL. In Proceedings of the 6th International Conference Interactive Theorem Proving. Christian Urban and Xingyuan Zhang (Eds.), Lecture Notes in Computer Science, Vol. 9236, Springer, 253-269. DOI:https://doi.org/10.1007/978-3-319-22102-1_17

[21] Peter Lammich. 2016. Refinement based verification of imperative data structures. In Proceedings of the 5th ACM SIGPLAN Conference on Certified Programs and Proofs, Jeremy Avigad and Adam Chlipala (Eds.). ACM, 27-36. DOI:https: //doi.org/10.1145/2854065.2854067

[22] Peter Lammich. 2019. Generating verified LLVM from Isabelle/HOL. In Proceedings of the 10th International Conference on Interactive Theorem Proving, John Harrison, John O'Leary, and Andrew Tolmach (Eds.). Schloss Dagstuhl - LeibnizZentrum für Informatik, 22:1-22:19. DOI:https://doi.org/10.4230/LIPIcs.ITP.2019.22

[23] Peter Lammich. 2019. Refinement to Imperative HOL. Fournal of Automated Reasoning 62, 4 (2019), 481-503. DOI:https: //doi.org/10.1007/s10817-017-9437-1

[24] Peter Lammich. 2020. Efficient verified implementation of introsort and pdqsort. In Proceedings of the IFCAR 2020 Nicolas Peltier and Viorica Sofronie-Stokkermans (Eds.), Lecture Notes in Computer Science, Vol. 12167, Springer, 307-323. DOI:https://doi.org/10.1007/978-3-030-51054-1_18

[25] Peter Lammich and Rene Meis. 2012. A separation logic framework for Imperative HOL. Archive of Formal Proofs (Nov. 2012). Retrieved from http://isa-afp.org/entries/Separation_Logic_Imperative_HOL.html, Formal proof development.

[26] Peter Lammich and Thomas Tuerk. 2012. Applying data refinement for monadic programs to Hopcroft's algorithm. In Proceedings of the 3rd International Conference on Interactive Theorem Proving. Lennart Beringer and Amy P. Felty (Eds.), Lecture Notes in Computer Science, Vol. 7406, Springer, 166-182. DOI:https://doi.org/10.1007/978-3-642-32347-8_12

[27] libc++ [n.d.]. "libc++" C++ Standard Library. Retrieved 20. May 2022 from https://releases.llvm.org/14.0.0/projects/ libcxx/docs/.

[28] Adrián Löwenberg Casas. 2019. Proof af the Amortized time complexity of an efficient Union-Find data structure in Isabelle/HOL. BS Thesis. Technical University of Munich.

[29] Glen Mével, Jacques-Henri Jourdan, and François Pottier. 2019. Time credits and time receipts in Iris. In Proceedings of the 28th European Symposium on Programming Languages and Systems, Luís Caires (Ed.), Lecture Notes in Computer Science, Vol. 11423, Springer, 3-29. DOI:https://doi.org/10.1007/978-3-030-17184-1_1

[30] David R. Musser. 1997. Introspective sorting and selection algorithms. Software Practice and Experience 27, 8 (1997), 983-993.

[31] Tobias Nipkow, Manuel Eberl, and Maximilian P. L. Haslbeck. 2020. Verified textbook algorithms - a biased survey. In Proceedings of the 18th International Symposium on Automated Technology for Verification and Analysis, Dang Van Hung and Oleg Sokolsky (Eds.), Lecture Notes in Computer Science, Vol. 12302, Springer, 25-53. D0I:https://doi.org/ 10.1007/978-3-030-59152-6_2

[32] Vineet Rajani. 2020. A type-theory for higher-order amortized analysis. Ph.D. Dissertation. Saarland University, Saarbrücken, Germany. Retrieved from https://publikationen.sulb.uni-saarland.de/handle/20.500.11880/29104.

[33] Vineet Rajani, Marco Gaboardi, Deepak Garg, and Jan Hoffmann. 2021. A unifying type-theory for higher-order (amortized) cost analysis. Proceedings of the ACM Programming Languages 5, POPL (2021), 1-28. DOI:https://doi.org/10.1145/ 3434308

[34] Philip Wadler. 1989. Theorems for free!. In Proceedings of the 4th International Conference on Functional Programming Languages and Computer Architecture, Joseph E. Stoy (Ed.). ACM, 347-359. DOI:https://doi.org/10.1145/99370.99404

[35] Philip Wadler. 1990. Comprehending monads. In Proceedings of the 1990 ACM Conference on LISP and Functional Programming. Association for Computing Machinery, New York, NY, 61-78. DOI:https://doi.org/10.1145/91556.91592

[36] Peng Wang, Di Wang, and Adam Chlipala. 2017. TiML: A functional language for practical complexity analysis with invariants. Proceedings of the ACM Programing Languages 1, OOPSLA (2017), 79:1-79:26. DOI:https://doi.org/10.1145/ 3133903

[37] Bohua Zhan and Maximilian P. L. Haslbeck. 2018. Verifying asymptotic time complexity of imperative programs in Isabelle. In Proceedings of the 9th International Joint Conference on Automated Reasoning, Didier Galmiche, Stephan Schulz, and Roberto Sebastiani (Eds.), Lecture Notes in Computer Science, Vol. 10900, Springer, 532-548. DOI:https: //doi.org/10.1007/978-3-319-94205-6_35

Received April 2021; revised July 2021; accepted August 2021 